\title{
Generalized Pattern Avoidance Condition for the Wreath Product of Cyclic Groups with Symmetric Groups
}

\author{
Sergey Kitaev, ${ }^{1}$ Jeffrey Remmel, ${ }^{2}$ and Manda Riehl ${ }^{3}$ \\ ${ }^{1}$ Department of Computer and Information Sceinces, University of Strathclyde Glasgow, Livingstone Tower, \\ 26 Richmond Street, Glasgow G1 1 XH, UK \\ ${ }^{2}$ Department of Mathematics, University of California, San Diego, La Jolla, CA 92093, USA \\ ${ }^{3}$ Department of Mathematics, University of Wisconsin Eau Claire, Eau Claire, WI 54702-4004, USA
}

Correspondence should be addressed to Sergey Kitaev; sergey.kitaev@gmail.com

Received 30 September 2012; Accepted 17 October 2012

Academic Editors: Y. Simsek and Y. Zhang

Copyright (C) 2013 Sergey Kitaev et al. This is an open access article distributed under the Creative Commons Attribution License, which permits unrestricted use, distribution, and reproduction in any medium, provided the original work is properly cited.

We continue the study of the generalized pattern avoidance condition for $C_{k} 2 S_{n}$, the wreath product of the cyclic group $C_{k}$ with the symmetric group $S_{n}$, initiated in the work by Kitaev et al., In press. Among our results, there are a number of (multivariable) generating functions both for consecutive and nonconsecutive patterns, as well as a bijective proof for a new sequence counted by the Catalan numbers.

\section{Introduction}

The goal of this paper is to continue the study of patternmatching conditions on the wreath product $C_{k}>S_{n}$ of the cyclic group $C_{k}$ and the symmetric group $S_{n}$ initiated in [1]. $C_{k} 2 S_{n}$ is the group of $k^{n} n$ ! signed permutations where there are $k$ signs, $1=\omega^{0}, \omega, \omega^{2}, \ldots, \omega^{k-1}$, where $\omega$ is a primitive $k$ th root of unity. We can think of the elements $C_{k} 2 S_{n}$ as pairs $\gamma=(\sigma, \epsilon)$ where $\sigma=\sigma_{1} \cdots \sigma_{n} \in S_{n}$ and $\epsilon=\epsilon_{1} \cdots \epsilon_{n} \in\left\{1, \omega, \ldots, \omega^{k-1}\right\}^{n}$. For ease of notation, if $\epsilon=$ $\left(\omega^{w_{1}}, \omega^{w_{2}}, \ldots, \omega^{w_{n}}\right)$ where $w_{i} \in\{0, \ldots, k-1\}$ for $i=1, \ldots, n$, then we simply write $\gamma=(\sigma, w)$ where $w=w_{1} w_{2} \cdots w_{n}$. Moreover, we think of the elements of $w=w_{1} w_{2} \cdots w_{n}$ as the colors of the corresponding elements of the underlying permutation $\sigma$.

We define a concept for matchings in words over a finite alphabet $[k]=\{0,1, \ldots, k-1\}$. Given a word $w=w_{1} \cdots w_{n} \in$ $[k]^{n}$, let red $(w)$ be the word found by replacing the $i$ th largest integer that appears in $w$ by $i-1$. For example, if $w=$

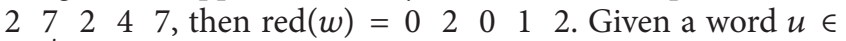
$[k]^{j}$ such that $\operatorname{red}(u)=u$, we say that a word $w \in[k]^{n}$ has a $u$-match starting at position $i$ provided $\operatorname{red}\left(w_{i} \cdots w_{i+j-1}\right)=u$. Let $u$-mch $(w)$ be the number of $u$-matches in the word $w$.
Similarly, we say that $u$ occurs in a word $w$ if there exist $1 \leq i_{1}<\cdots<i_{j} \leq n$ such that $\operatorname{red}\left(w_{i_{1}} \cdots w_{i_{j}}\right)=u$. We say that $w$ avoids $u$ if there are no occurrences of $u$ in $w$.

There are a number of papers on pattern matching and pattern avoidance in $C_{k}<S_{n}$ [2-5]. We now present a selection of the previously studied definitions for pattern matching and avoidance, so that the reader may see how ours differs from and/or extends those in the literature. For example, the following pattern matching condition was studied in $[3-5]$.

Definition 1. Let $(\tau, u) \in C_{k}<S_{j}, \Upsilon$ be a subset of $C_{k} 2 S_{j}$, and $(\sigma, w) \in C_{k} 2 S_{n}$.

(1) One says that $(\sigma, w)$ has an exact occurrence of $(\tau, u)$ (resp., $\Upsilon$ ) if there are $1 \leq i_{1}<i_{2}<\cdots<i_{j} \leq n$ such that $\left(\operatorname{red}\left(\sigma_{i_{1}} \cdots \sigma_{i_{j}}\right), w_{i_{1}} \cdots w_{i_{j}}\right)=(\tau, u)$ (resp., $\left.\left(\operatorname{red}\left(\sigma_{i_{1}} \cdots \sigma_{i_{j}}\right), w_{i_{1}} \cdots w_{i_{j}}\right) \in \Upsilon\right)$.

(2) One says that $(\sigma, w) \in C_{k} \geq S_{n}$ avoids an exact occurrence of $(\tau, u)$ (resp., $Y$ ) if there are no exact occurrences of $(\tau, u)$ (resp., $\Upsilon$ ) in $(\sigma, w)$.

(3) One says that there is an exact $(\tau, u)$-match in $(\sigma, w)$ starting at position $i$ (resp., exact 
$\Upsilon$-match in $(\sigma, w)$ starting at position $i)$ if $\left(\operatorname{red}\left(\sigma_{i} \sigma_{i+1} \cdots \sigma_{i+j-1}\right), w_{i} w_{i+1} \cdots w_{i+j-1}\right)=(\tau, u)$ (resp., $\left(\operatorname{red}\left(\sigma_{i} \sigma_{i+1} \cdots \sigma_{i+j-1}\right), w_{i} w_{i+1} \cdots w_{i+j-1}\right) \in \Upsilon$ ).

That is, an exact occurrence or an exact match of $(\tau, u) \epsilon$ $C_{k}<S_{j}$ in an element $(\sigma, w) \in C_{k}<S_{n}$ is just an ordinary occurrence or match of $\tau$ in $\sigma$ where the corresponding signs agree exactly. For example, Mansour [4] proved via recursion that, for any $(\tau, u) \in C_{k}<S_{2}$, the number of elements in $C_{k}<S_{n}$ which avoid exact occurrences of $(\tau, u)$ is $\sum_{j=0}^{n} j !(k-1)^{j}\left(\begin{array}{c}n \\ j\end{array}\right)^{2}$. This generalized a result of Simion [6] who proved the same result for the hyperoctahedral group $C_{2}$ \& $S_{n}$. Similarly, Mansour and West [5] determined the number of permutations in $C_{2} \geqslant S_{n}$ that avoid all possible exact occurrences of 2 or 3 element sets of patterns from $C_{2}$ \& $S_{2}$. For example, let $K_{n}^{1}$ be the number of $(\sigma, \epsilon) \epsilon$ $C_{2}, S_{n}$ that avoid all exact occurrences of the patterns in the set $\left\{\left(\begin{array}{lll}1 & 2,0 & 0\end{array}\right),\left(\begin{array}{lll}1 & 2,0 & 1\end{array}\right),\left(\begin{array}{lll}2 & 1,1 & 0\end{array}\right)\right\}, K_{n}^{2}$ the number of $(\sigma, \epsilon) \in C_{2}$ ? $S_{n}$ that avoid all exact occurrences of the patterns in the set $\left\{\left(\begin{array}{lll}1 & 2,0 & 1\end{array}\right),\left(\begin{array}{lll}1 & 2,1 & 0\end{array}\right),\left(\begin{array}{lll}2 & 1,0 & 1\end{array}\right)\right\}$, and $K_{n}^{3}$ the number of $(\sigma, \epsilon) \in C_{2} \geqslant S_{n}$ that avoid all exact occurrences of the patterns in the set $\left\{\left(\begin{array}{lll}1 & 2,0 & 0\end{array}\right),\left(\begin{array}{llll}1 & 2,0 & 1\end{array}\right),\left(\begin{array}{lll}2 & 1,0 & 0\end{array}\right)\right.$. Then Mansour and West [5] proved that

$$
\begin{gathered}
K_{n}^{1}=F_{2 n+1}, \\
K_{n}^{2}=n ! \sum_{j=0}^{n}\left(\begin{array}{c}
n \\
j
\end{array}\right)^{-1}, \\
K_{n}^{3}=n !+n ! \sum_{j=1}^{n} \frac{1}{j},
\end{gathered}
$$

where $F_{n}$ is the $n$th Fibonacci number.

An alternative matching condition arises when we drop the requirement of the exact matching of signs and replace it by the condition that the two sequences of signs match in the sense of words described above. That is, we shall consider the following matching conditions.

Definition 2. Let $(\tau, u) \in C_{k}>S_{j}$ where $\operatorname{red}(u)=u, \Upsilon$ a subset of $C_{k}>S_{j}$ where for all $(\tau, u) \in \Upsilon$, $\operatorname{red}(u)=u$, and $(\sigma, w) \in$ $C_{k}<S_{n}$.

(1) One says that $(\sigma, w)$ has an occurrence of $(\tau, u)$ (resp., $\Upsilon)$ if there are $1 \leq i_{1}<i_{2}<\cdots<i_{j} \leq n$ such that $\left(\operatorname{red}\left(\sigma_{i_{1}} \cdots \sigma_{i_{j}}\right), \operatorname{red}\left(w_{i_{1}} \cdots w_{i_{j}}\right)\right)=(\tau, u)$ (resp., $\left.\left(\operatorname{red}\left(\sigma_{i_{1}} \cdots \sigma_{i_{j}}\right), \operatorname{red}\left(w_{i_{1}} \cdots w_{i_{j}}\right)\right) \in \Upsilon\right)$.

(2) One says that $(\sigma, w) \in C_{k} 2 S_{n}$ avoids $(\tau, u)$ (rep., $\Upsilon$ ) if there are no occurrences of $(\tau, u)$ (resp., $\Upsilon)$ in $(\sigma, w)$.

(3) One says that there is a $(\tau, u)$-match in $(\sigma, w)$ starting at position $i$ (resp., $\Upsilon$ match in $(\sigma, w)$ starting at position $i)$ if $\left(\operatorname{red}\left(\sigma_{i} \sigma_{i+1} \cdots \sigma_{i+j-1}\right), \operatorname{red}\left(w_{i} w_{i+1} \cdots w_{i+j-1}\right)\right)=(\tau, u)$ (resp., $\left(\operatorname{red}\left(\sigma_{i} \sigma_{i+1} \cdots \sigma_{i+j-1}\right), \operatorname{red}\left(w_{i} w_{i+1} \cdots w_{i+j-1}\right)\right) \epsilon$ $\Upsilon)$.
For example, suppose that $(\tau, u)=\left(\begin{array}{lll}1 & 2,0 & 0\end{array}\right)$ and $(\sigma, w)=$ $\left(\begin{array}{lllllll}1 & 3 & 2 & 4,1 & 2 & 2 & 2\end{array}\right)$. Then there are no exact occurrences or exact matches of $(\tau, u)$ in $(\sigma, w)$. However, there are two occurrences of $(\tau, u)$, one in positions 2 and 4 and one in positions 3 and 4 . Thus there are two occurrences of $(\tau, u)$ in $(\sigma, w)$ and there is a $(\tau, u)$-match in $(\sigma, w)$ starting at position 3.

Finally, a more general-matching condition which generalizes both occurrences and matches and exact occurrences and exact matches in $C_{k}<S_{n}$ was introduced in [1].

Definition 3. Let $(\tau, u, \vec{A}) \in C_{k}>S_{j}$, $\Upsilon$ be a subset of $C_{k}>S_{j}$, $\vec{A}=\left(A_{1}, \ldots, A_{j}\right)$ be a sequence of subsets of $[k]$, and $(\sigma, w) \epsilon$ $C_{k} \prec S_{n}$.

(1) One says that $(\sigma, w)$ has an occurrence of $(\tau, u, \vec{A})$ (resp., $(\Upsilon, \vec{A}))$ if there are $1 \leq i_{1}<i_{2}<\cdots<$ $i_{j} \leq n$ such that $\left(\operatorname{red}\left(\sigma_{i_{1}} \cdots \sigma_{i_{j}}\right), \operatorname{red}\left(w_{i_{1}} \cdots w_{i_{j}}\right)\right)=$ $(\tau, \operatorname{red}(u))$ (resp., $\left(\operatorname{red}\left(\sigma_{i_{1}} \cdots \sigma_{i_{j}}\right), \operatorname{red}\left(w_{i_{1}} \cdots w_{i_{j}}\right)\right)$ is equal to $(\tau, \operatorname{red}(u))$ for some $(\tau, u)$ in $\Upsilon)$ and $w_{i_{s}} \in A_{s}$ for $s=1, \ldots, j$.

(2) One says that $(\sigma, w) \in C_{k} 2 S_{n}$ avoids $(\tau, u, \vec{A})$ (resp., $(\Upsilon, \vec{A}))$ if there are no occurrences of $(\tau, u)$ (resp., $\Upsilon$ ) in $(\sigma, w)$.

(3) One says that there is a $(\tau, u, \vec{A})$-match in $(\sigma, w)$ starting at position $i$ (resp., $(\Upsilon, \vec{A})$-match in $(\sigma, w)$ starting at position $i)$ if $\left(\operatorname{red}\left(\sigma_{i} \sigma_{i+1} \cdots \sigma_{i+j-1}\right)\right.$, $\left.\operatorname{red}\left(w_{i} w_{i+1} \cdots w_{i+j-1}\right)\right)=(\tau, \operatorname{red}(u))$ (resp., $\left(\operatorname{red}\left(\sigma_{i} \sigma_{i+1} \cdots \sigma_{i+j-1}\right), \operatorname{red}\left(w_{i} w_{i+1} \cdots w_{i+j-1}\right)\right)$ is equal to $(\tau, \operatorname{red}(u))$ for some $(\tau, u)$ in $\Upsilon)$ and $w_{i+s} \in A_{s+1}$ for $s=0, \ldots, j-1$.

Thus a $(\tau, u, \vec{A})$-occurrence (or a $(\tau, u, \vec{A})$-match) where $u=u_{1} \cdots u_{j}$ and $\vec{A}=\left(\left\{u_{1}\right\}, \ldots,\left\{u_{j}\right\}\right)$ is just an exact occurrence (resp., an exact match) of $(\tau, u)$. Similarly, a $(\tau, u, \vec{A})$ occurrence (or a $(\tau, u, \vec{A})$-match) where $u=u_{1} \cdots u_{j}$, $\operatorname{red}(u)=u$, and $\vec{A}=\left(A_{1}, \ldots, A_{j}\right)$ such that $A_{i}=$ [k] for $i=1, \ldots, j$ is just an occurrence (or match) of $(\tau, u)$.

Suppose we are given $(\tau, u) \in C_{k}$ ? $S_{j}, \Upsilon \subseteq C_{k}$ ? $S_{j}, \vec{A}=\left(A_{1}, \ldots, A_{j}\right)$ where $A_{i} \subseteq[k]$ for $i=$ $1, \ldots, j$, and $(\sigma, w) \in C_{k}, S_{n}$. We let $(\tau, u)-\operatorname{mch}((\sigma, w))$ (resp., $(\tau, u)-\operatorname{Emch}((\sigma, w)))$ denote the number of $(\tau, u)$ matches (resp., exact $(\tau, u)$-matches) in $(\sigma, w)$. We let $\Upsilon$ - $\operatorname{mch}((\sigma, w))$ (resp., $\Upsilon$-Emch $((\sigma, w)))$ denote the number $\Upsilon$-matches (resp., exact $\Upsilon$-matches) in $(\sigma, w)$. We let $(\tau, u, \vec{A})-\operatorname{mch}((\sigma, w))$ be the number of $(\tau, u, \vec{A})$-matches in $(\sigma, w)$ and $(\Upsilon, \vec{A})-\operatorname{mch}((\sigma, w))$ the number of $(\Upsilon, \vec{A})$-matches in $(\sigma, w)$.

The main result of [1] was to derive a generating function for the distribution of $(\tau, u, \vec{A})$-matches where $(\tau, u) \in C_{k}$ is any element of $C_{k}<S_{2}$. To state the main result of [1], we need to define some notation. First we define the $p, q$-analogues of 
$n, n !,\left(\begin{array}{l}n \\ k\end{array}\right)$, and $\left(\begin{array}{c}n \\ a_{1}, \ldots, a_{m}\end{array}\right)$ by

$$
\begin{aligned}
& {[n]_{p, q}=\frac{p^{n}-q^{n}}{p-q}=p^{n-1}+p^{n-2} q} \\
& +\cdots+p q^{n-2}+q^{n-1}, \\
& {[n]_{p, q} !=[n]_{p, q}[n-1]_{p, q} \cdots[2]_{p, q}[1]_{p, q},} \\
& {\left[\begin{array}{l}
n \\
k
\end{array}\right]_{p, q}=\frac{[n]_{p, q} !}{[k]_{p, q} ![n-k]_{p, q} !},} \\
& {\left[\begin{array}{c}
n \\
a_{1}, \ldots, a_{m}
\end{array}\right]_{p, q}=\frac{[n]_{p, q} !}{\left[a_{1}\right]_{p, q} ! \cdots\left[a_{m}\right]_{p, q} !},}
\end{aligned}
$$

respectively. We define the $q$-analogs of $n, n !,\left(\begin{array}{l}n \\ k\end{array}\right)$, and $\left(\begin{array}{c}n \\ a_{1}, \ldots, a_{m}\end{array}\right)$ by $[n]_{1, q},[n]_{1, q} !,\left[\begin{array}{l}n \\ k\end{array}\right]_{1, q}$, and $\left[a_{1}, \ldots, a_{m}\right]_{1, q}$, respectively.

Next suppose that $\Upsilon \subseteq C_{k}>S_{2}$ and $\vec{A}=\left(A_{1}, A_{2}\right)$ where $A_{1}, A_{2} \subseteq[k]$. Then we will say that $(\sigma, w) \in C_{k} ? S_{n}$ is a maximum packing for $(\Upsilon, \vec{A})$ if $(\sigma, w)$ has $(\Upsilon, \vec{A})$-matches starting at positions $1,2, \ldots, n-1$. We let $\mathscr{M}_{(\Upsilon, \vec{A}), n}$ denote the set $(\sigma, w) \in C_{k}, S_{n}$ of maximum packings for $(\Upsilon, \vec{A})$. Given any word $w=w_{1} \cdots w_{n} \in[k]^{n}$, we let $z(w)=\prod_{i=1}^{n} z_{w_{i}}$. For any $\sigma=\sigma_{1} \cdots \sigma_{n} \in S_{n}$, we let $\operatorname{inv}(\sigma)(\operatorname{resp}$., $\operatorname{coinv}(\sigma))$ equal the number of pairs $(i, j)$ such that $1 \leq i<j \leq n$ and $\sigma_{i}>\sigma_{j}\left(\right.$ resp., $\left.\sigma_{i}<\sigma_{j}\right)$. We then define

$$
\begin{aligned}
& m p_{(\Upsilon, \vec{A}), n}\left(p, q ; z_{0}, \ldots, z_{k-1}\right) \\
& =\sum_{(\sigma, w) \in \mathscr{M}_{(\Upsilon, \vec{A}), n}} p^{\operatorname{coinv}(\sigma)} q^{\operatorname{inv}(\sigma)} z(w) .
\end{aligned}
$$

We will also be interested in the specializations

$$
\begin{gathered}
m P_{(\Upsilon, \vec{A}), n}(p, q ; 1, \ldots, 1) \\
=\sum_{(\sigma, w) \in M \mathscr{P}_{(Y, \vec{A}), n}} p^{\operatorname{coinv}(\sigma)} q^{\operatorname{inv}(\sigma)}, \\
m P_{(\Upsilon \vec{A}), n}\left(p, q ; 1, r, \ldots, r^{k-1}\right) \\
=\sum_{(\sigma, w) \in \mathscr{M P}_{(\Upsilon, \vec{A}), n}} p^{\operatorname{coinv}(\sigma)} q^{\operatorname{inv}(\sigma)} r^{\|w\|},
\end{gathered}
$$

where for any word $w=w_{1} \cdots w_{n} \in[k]^{n},\|w\|=w_{1}+$ $\cdots+w_{n}$. In the special case where $\Upsilon=\{(\tau, u)\}$ where $(\tau, u) \in C_{k} 2 S_{2}$, we shall denote $m p_{(\Upsilon, \vec{A}), n}\left(p, q ; z_{0}, \ldots, z_{k-1}\right)$ as $m p_{(\tau, u, \vec{A}), n}\left(p, q, z_{0}, \ldots, z_{k-1}\right)$.
Then Kitaev et al. [1] proved that, for any $\Upsilon \subseteq C_{k} 2 S_{2}$ and $\vec{A}=\left(A_{1}, A_{2}\right)$ where $A_{1}, A_{2} \subseteq[k]$,

$$
\begin{gathered}
\sum_{n \geq 0} \frac{t^{n}}{[n]_{p, q} !} \sum_{(\sigma, w) \in C_{k} S_{n}} p^{\operatorname{coinv}(\sigma)} q^{\operatorname{inv}(\sigma)} x^{(\Upsilon, \vec{A})-\operatorname{mch}((\sigma, w))} z(w) \\
=\left(1-\left(\left(z_{0}+\cdots+z_{k-1}\right) t+\sum_{n \geq 2} \frac{t^{n}}{[n]_{p, q} !}(x-1)^{n-1}\right.\right. \\
\left.\left.m p_{(\Upsilon, \vec{A}), n}\left(p, q ; z_{0}, \ldots, z_{k-1}\right)\right)\right)^{-1}
\end{gathered}
$$

Duane and Remmel [7] gave the generating function for the number of $(\tau, u)$-matches and exact $(\tau, u)$-matches for a certain collection of $(\tau, u) \in C_{j}>S_{n}$ where $j \geq 3$ are called minimal overlapping patterns. Given a word $u \in\{0,1, \ldots, k-$ $1\}^{j}$ such that $\operatorname{red}(u)=u$ and $\tau \in S_{j}$, Duane and Remmel say that $(\tau, u)$ has the $C_{k}>S_{n}$-minimal overlapping property if the smallest $i$ such that there exists a $(\sigma, w) \in C_{k}$ ? $S_{i}$ with $(\tau, u)-\operatorname{mch}((\sigma, w))=2$ is $2 j-1$. This means that in a $k$ colored permutation $(\sigma, w)$, two $(\tau, u)$-matches can share at most one pair of letters, and this pair of letters must occur at the end of the first $(\tau, u)$-match and at the start of the second $(\tau, u)$-match. Similarly, we say that $(\tau, u)$ has the $C_{k}<S_{n}$-exact match minimal overlapping property if the smallest $i$ such that there exists a $(\sigma, w) \in C_{k} 2 S_{i}$ with $(\tau, u)-\operatorname{Emch}((\sigma, w))=2$ is $2 j-1$. Now if $(\tau, u)$ has the $C_{k}>S_{n}$-minimal overlapping property, then the shortest $k$-colored permutations $(\sigma, w)$, such that $(\tau, u)-\operatorname{mch}((\sigma, w))=n$ have length $n(j-1)+1$. We let $\mathscr{M}_{(\tau, u), n(j-1)+1}^{k}$ equal the set of $k$-colored permutations, $(\sigma, w) \in C_{k} ? S_{n(j-1)+1}$, such that $(\tau, u)-\operatorname{mch}(\sigma, w)=n$. We refer to the $k$-colored permutations in $\mathscr{M}_{(\tau, u), n(j-1)+1}^{k}$ as maximum packings for $(\tau, u)$. We let

$$
\begin{gathered}
m p_{(\tau, u), n(j-1)+1}^{k}=\left|\mathscr{M}_{(\tau, u), n(j-1)+1}^{k}\right|, \\
m p_{(\tau, u), n(j-1)+1}^{k}(p, q, r) \\
=\sum_{(\sigma, w) \in \mathscr{M}_{\mathscr{P}_{(\tau, u), n(j-1)+1}^{k}}^{k}} p^{\operatorname{coinv}(\sigma)} q^{\operatorname{inv}(\sigma)} r^{\sum w}, \\
m p_{(\tau, u), n(j-1)+1}^{k}\left(p, q ; z_{0}, \ldots, z_{k-1}\right) \\
=\sum_{(\sigma, w) \in \mathscr{M} \mathscr{P}_{(\tau, u), n(j-1)+1}^{k}} p^{\operatorname{coinv}(\sigma)} q^{\operatorname{inv}(\sigma)} z(w) .
\end{gathered}
$$

Similarly, we let $\mathscr{E} \mathscr{M} \mathscr{P}_{(\tau, u), n(j-1)+1}^{k}$ denote the set of $k$ colored permutations, $(\sigma, w) \in C_{k}<S_{n(j-1)+1}$, such that 
$(\tau, u)-\operatorname{Emch}(\sigma, w)=n$, and refer to the $k$-colored permutations in $\mathscr{E} \mathscr{M}_{\mathscr{P}^{k}(\tau), n(j-1)+1}^{k}$ as exact match maximum packings for $(\tau, u)$. We let

$$
\begin{gathered}
\operatorname{emp}_{(\tau, u), n(j-1)+1}^{k}=\mid \mathscr{E} \mathscr{M}_{(\tau, u), n(j-1)+1} \mathscr{P}^{k} \\
e m p_{(\tau, u), n(j-1)+1}^{k}(p, q, r) \\
=\sum_{(\sigma, w) \in \mathscr{C} \mathscr{M}_{(\tau, u), n(j-1)+1}^{k}} p^{\operatorname{coinv}(\sigma)} q^{\operatorname{inv}(\sigma)} r^{\sum w}, \\
e m p_{(\tau, u), n(j-1)+1}^{k}\left(p, q ; z_{0}, \ldots, z_{k-1}\right) \\
=\sum_{(\sigma, w) \in \mathscr{E} \mathscr{M} \mathscr{P}_{(\tau, u), n(j-1)+1}^{k}} p^{\operatorname{coinv}(\sigma)} q^{\operatorname{inv}(\sigma)} z(w) .
\end{gathered}
$$

Duane and Remmel [7] proved that (I) if $\operatorname{red}(u)=u$ and $(\tau, u)$ has the $C_{k}$ > $S_{n}$-minimal overlapping property, then

$$
\begin{aligned}
\sum_{n \geq 0} \frac{t^{n}}{n !} \sum_{(\sigma, w) \in C_{k} \text { lS }} x^{(\tau, u)-\operatorname{mch}(\sigma, u)} p^{\operatorname{coinv}(\sigma)} q^{\operatorname{inv}(\sigma)} z(w) \\
=1 /\left(1-\left(\left(z_{0}+\cdots+z_{k-1}\right) t\right.\right. \\
+\sum_{n \geq 1} \frac{t^{n(j-1)+1}}{[n(j-1)+1]_{p, q} !}(x-1)^{n} \\
\left.\left.\quad \times m p_{(\tau, u), n(j-1)+1}^{k}\left(p, q ; z_{0}, \ldots, z_{k-1}\right)\right)\right),
\end{aligned}
$$

and (II) if $(\tau, u) \in C_{k}>S_{j}$ has the $C_{k} 2 S_{n}$-exact match minimal overlapping property, then

$$
\begin{aligned}
& \sum_{n \geq 0} \frac{t^{n}}{n !} \sum_{(\sigma, w) \in C_{k} S_{n}} x^{(\tau, u)-\operatorname{Emch}(\sigma, u)} p^{\operatorname{coinv}(\sigma)} q^{\operatorname{inv}(\sigma)} z(w) \\
&=1 /\left(1-\left(\left(z_{0}+\cdots+z_{k-1}\right) t\right.\right. \\
&+\sum_{n \geq 1} \frac{t^{n(j-1)+1}}{[n(j-1)+1]_{q} !}(x-1)^{n} \\
&\left.\left.\quad \operatorname{eemp}_{(\tau, u), n(j-1)+1}^{k}\left(p, q, z_{0}, \ldots, z_{k-1}\right)\right)\right) .
\end{aligned}
$$

Duane and Remmel's proof of (I) and (II) works equally well for $(\Upsilon, \vec{A})$-matches.

The main goal of this paper is to prove a number of results on the number of $(\tau, u, \vec{A})$-avoiding elements of $C_{k}>S_{n}$ and to find a generating function for the number of $(\tau, u, \vec{A})$ matches for certain patterns which do not have the minimal overlapping property. That is, in Section 2, we shall prove a number of results about the number of $(\tau, u, \vec{A})$-avoiding elements of $C_{k}$ ? $S_{n}$ where $(\tau, u) \in C_{k}>S_{2}$. In Section 3, we prove a number of results on the number of $(\Upsilon, \vec{A})$-avoiding elements of $C_{k}<S_{n}$ and for certain subsets $\Upsilon$ of $C_{k}<S_{2}$.
Finally, in Section 4, we will prove an analog of a theorem of Mendes and Remmel which gave a generating function by the number of descents for the set of elements of $S_{n}$ that had no $(j(j-1) \cdots 1)$-matches.

\section{2. $(\tau, u, \vec{A})$-Avoiding Patterns}

Given $(\tau, u) \in C_{k}<S_{j}$ and $\vec{A}=\left(A_{1}, \ldots, A_{j}\right)$ where $A_{i} \subseteq[k]$ for $i=1, \ldots, j$, let $A v^{(\tau, u, \vec{A})}$ denote the number of $(\sigma, w) \in$ $C_{k}$ ? $S_{n}$ which avoid $(\tau, u, \vec{A})$. In the special case where $A_{i}=$ $[k]$ for $i=1, \ldots, j$, we shall denote $A v^{(\tau, u, \vec{A})}$ as simply $A v^{(\tau, u)}$. Thus $A v^{(\tau, u)}$ is the number $(\sigma, w) \in C_{k} 2 S_{n}$ which avoid $(\tau, u)$. In this case, we shall find formulas for $A v^{(\tau, u, \vec{A})}$ or $A v^{(\tau, u)}$ where $(\tau, u) \in C_{k}$ ? $S_{2}$.

There are a number of natural maps for which the distribution of $(\tau, u, \vec{A})$-occurrences and $(\tau, u, \vec{A})$-matches remain invariant. That is, for any $\sigma=\sigma_{1} \cdots \sigma_{n} \in S_{n}$, we define the reverse of $\sigma, \sigma^{r}$ and the complement of $\sigma, \sigma^{c}$, respectively, by

$$
\begin{gathered}
\sigma^{r}=\sigma_{n} \sigma_{n-1} \cdots \sigma_{1}, \\
\sigma^{c}=\left(n+1-\sigma_{1}\right) \cdots\left(n+1-\sigma_{n}\right) .
\end{gathered}
$$

If $w=w_{1} \cdots w_{n} \in[k]^{n}$, then we define the reverse of $w, w^{r}$ and the complement of $w$ relative to $[k], w^{(c, k)}$, by

$$
\begin{gathered}
w^{r}=w_{n} w_{n-1} \cdots w_{1}, \\
w^{(c, k)}=\left(k-1-w_{1}\right) \cdots\left(k-1-w_{n}\right) .
\end{gathered}
$$

If $A=\left\{a_{1}, \ldots, a_{s}\right\} \subseteq[k]$, then we define $A^{(c, k)}=\{k-1-$ $\left.a_{1}, \ldots, k-1-a_{s}\right\}$. Then if $\vec{A}=\left(A_{1}, \ldots, A_{j}\right)$ where $A_{i} \subseteq[k]$, for $i=1, \ldots, j$, then we define

$$
\begin{aligned}
& \vec{A}^{r}=\left(A_{j}, A_{j-1}, \ldots, A_{1}\right), \\
& \vec{A}^{(c, k)}=\left(A_{1}^{c, k}, \ldots, A_{j}^{c, k}\right) .
\end{aligned}
$$

Then consider the maps $\phi_{a, b}: C_{k}<S_{n} \rightarrow C_{k}<S_{n}$ where $\phi_{a, b}((\sigma, w))=\left(\sigma^{a}, w^{b}\right)$ for $a, b \in\{i, r, c\}$ and $b \in\{i, r,(c, k)\}$ where $i$ is the identity map; that is, $\sigma^{i}=\sigma$ for all $\sigma \in S_{n}$, $w^{i}=w$ for all $w \in[k]^{n}$, and $\vec{A}^{i}=\vec{A}$ for all $\vec{A}=\left(A_{1}, \ldots, A_{n}\right)$ with $A_{i} \subseteq[k]$ for $i=1, \ldots, n$. It is easy to see that a $(\sigma, \tau)$ has a $(\tau, w, \vec{A})$-match or $(\tau, w, \vec{A})$-occurrence if and only if $\left(\sigma^{a}, w^{b}\right)$ has a $\left(\tau^{a}, u^{b}, \vec{A}^{b}\right)$-occurrence or $\left(\tau^{a}, u^{b}, \vec{A}^{b}\right)$-match.

It follows that if $\operatorname{red}(u)=u$ and $(\tau, u) \in C_{k}>S_{2}$, then we need only to compute $A v^{(\tau, u)}$ for two patterns; namely, $(\tau, u)=\left(\begin{array}{lll}1 & 2,0 & 0\end{array}\right)$ and $(\tau, u)=\left(\begin{array}{lll}1 & 2,0 & 1\end{array}\right)$.

We start by considering the pattern $\left(\begin{array}{lll}1 & 2,0 & 0\end{array}\right)$.

Lemma 4. The number of elements in $C_{k}<S_{n}$ avoiding ( 12,0 0) is given by

$$
\sum_{\substack{i_{1}+\cdots+i_{k}=n \\
i_{1} \geq 0, \ldots, i_{k} \geq 0}}\left(\begin{array}{c}
n \\
i_{1}, \ldots, i_{k}
\end{array}\right)^{2} .
$$


Proof. We first observe that elements of different colors are independent in permutations avoiding ( $\left.\begin{array}{lll}1 & 2,0 & 0\end{array}\right)$, meaning that no two elements with different colors can form a prohibited configuration. Thus assuming we have $i_{j+1}$ elements of color $j, j=0, \ldots, k-1$, we can choose how to place these colors to form a word $w$ in $\left(\begin{array}{c}n \\ i_{1}, \ldots, i_{k}\end{array}\right)$ ways. Then we can choose the sets of elements $C_{0}, \ldots, C_{k-1}$ from $\{1, \ldots, n\}$ which will correspond to the colors $0, \ldots, k-1$ in $\sigma$ in $\left({ }_{i}, \ldots, i_{k}\right)$ ways. Finally, in order to construct a $(\sigma, w)$ which avoids the prohibited pattern, we must place the elements of $C_{i}$ in the positions which are colored by $i$ in decreasing order.

The proof of Lemma 4 suggests an obvious generalization for patterns of the form $\left(\tau, 0^{j}\right)$ where $\tau \in S_{j}$.

Theorem 5. Let $\tau \in S_{j}$. Then the number of elements in $C_{k}>S_{n}$ avoiding $\left(\tau, 0^{j}\right)$ is given by

$$
\sum_{\substack{i_{1}+\cdots+i_{k}=n \\
i_{1} \geq 0, \ldots, i_{k} \geq 0}} A_{i_{1}} A_{i_{2}} \cdots A_{i_{k}}\left(\begin{array}{c}
n \\
i_{1}, \ldots, i_{k}
\end{array}\right)^{2}
$$

where $A_{n}$ is the number of permutations in $S_{n}$ avoiding $\tau$.

Proof. The proof here is essentially the same as the proof of Theorem 4, except that we can place $i$ elements of a permutation in $C_{k} 2 S_{n}$ of the same color in any of $A_{i}$ ways.

It is well known that the number of $n$-permutations avoiding any pattern of length 3 is given by the $n$th Catalan number $C_{n}=(1 /(n+1))\left(\begin{array}{c}2 n \\ n\end{array}\right)$. As a corollary to Theorem 5 , we have that, for any pattern $\tau \in S_{3}$, the number of permutations in $C_{k}<S_{n}$ avoiding $(\tau, 0 \quad 0 \quad 0)$ is given by

$$
\sum_{\substack{i_{1}+\cdots+i_{k}=n \\
i_{1} \geq 0, \ldots, i_{k} \geq 0}} \frac{\left(\begin{array}{c}
2 i_{1} \\
i_{1}
\end{array}\right)\left(\begin{array}{c}
2 i_{2} \\
i_{2}
\end{array}\right) \cdots\left(\begin{array}{c}
2 i_{k} \\
i_{k}
\end{array}\right)}{\left(i_{1}+1\right)\left(i_{2}+1\right) \cdots\left(i_{k}+1\right)}\left(\begin{array}{c}
n \\
i_{1}, \ldots, i_{k}
\end{array}\right)^{2} .
$$

One can generalize Theorems 4 and 5 even further, by considering the distribution of patterns. Indeed, assuming that we know the number $A_{i, m}$ of $i$-permutations containing $m$ occurrences of a pattern $\tau \in S_{j}$, we can write down the number of permutations in $C_{k}<S_{n}$ with $m$ occurrences of $\left(\tau, 0^{j}\right)$ as

$$
\sum_{\substack{i_{1}+\cdots+i_{k}=n \\
m_{1}+\cdots+m_{k}=m \\
i_{1} \geq 0, \ldots, i_{k} \geq 0 \\
m_{1} \geq 0, \ldots, m_{k} \geq 0}} A_{i_{1}, m_{1}} A_{i_{2}, m_{2}} \cdots A_{i_{k}, m_{k}}\left(\begin{array}{c}
n \\
i_{1}, \ldots, i_{k}
\end{array}\right)^{2} .
$$

For example, the distribution of the occurrences of the pattern 12 is the same as the distribution of coinversions in permutations, so one can extract the numbers $A_{i, m}$ as the coefficients to $q^{m}$ in $\prod_{s=1}^{i}\left(1+q+\cdots+q^{s-1}\right)$ and substitute them in the last formula to get the distribution of the number of occurrences of (1 2,0 , 0 ) in $C_{k} 2 S_{n}$.

Next we consider the pattern $\left(\begin{array}{lll}1 & 2,0 & 1\end{array}\right)$ in the case where $k=2$.
The number of permutations in $C_{2} \geq S_{n}$ avoiding $\left(\begin{array}{lll}1 & 2,0 & 1\end{array}\right)$ is shown in [6, page 19] to be equal to $\sum_{j=0}^{n} j !\left(\begin{array}{c}n \\ j\end{array}\right)^{2}$. However, in Theorem 6 below we provide an independent derivation of the exponential generating function in this case.

Theorem 6. The exponential generating function for the number of elements in $C_{2}>S_{n}$ avoiding the pattern $\left(\begin{array}{lll}1 & 2,0 & 1\end{array}\right)$ is given by

$$
A(t)=\frac{e^{t /(1-t)}}{1-t} .
$$

Proof. Let $A_{n}=A v^{\left(\begin{array}{lll}1 & 2,0 & 1\end{array}\right)}$ and

$$
A(t)=\sum_{n \geq 0} A_{n} \frac{t^{n}}{n !} .
$$

If $(\sigma, w) \in C_{2}>S_{n+1}$ contains the element 1 colored by the color 1 , then there are no restrictions for placing this element, thereby giving $(n+1) A_{n}$ possibilities. On the other hand, if $(\sigma, w)$ contains the element 1 colored by the color 0 in position $i+1$ and $(\sigma, w)$ is to avoid ( $\left.\begin{array}{lll}1 & 2,0 & 1\end{array}\right)$, it must be the case that every element to the right of 1 is colored with the color 0 and these elements can be arranged in any of $(n-i)$ ! ways. Moreover, it immediately follows that no instance of occurrence of ( $\left.\begin{array}{lll}1 & 2,0 & 1\end{array}\right)$ exists where the first element is to the left of 1 and the second is to the right of 1 . Thus it follows that $(\sigma, w)$ avoids (1 2,0 1) if and only if there is no occurrence of $\left(\begin{array}{lll}1 & 2,0 & 1\end{array}\right)$ in $\left(\sigma_{1} \cdots \sigma_{i}, w_{1} \cdots w_{i}\right)$. Then in the case where 1 is colored by 0 and is in position $i+1$, we have $(n-i) !\left(\begin{array}{c}n \\ i\end{array}\right) A_{i}=n ! A_{i} / i$ ! possibilities where the binomial coefficient is responsible for choosing the elements to the left of 1 and placing the remaining elements to the right of 1 . To summarize, we obtain

$$
A_{n+1}=(n+1) A_{n}+n ! \sum_{i=0}^{n} \frac{A_{i}}{i !} .
$$

Multiplying both parts of the equation above by $t^{n} / n$ ! and summing over all $n \geq 0$, we have

$$
\begin{aligned}
\sum_{n \geq 0} A_{n+1} \frac{t^{n}}{n !}= & \sum_{n \geq 0} A_{n} \frac{t^{n}}{n !} \\
& +\sum_{n \geq 1} n A_{n} \frac{t^{n}}{n !}+\sum_{n \geq 0} t^{n} \sum_{i=0}^{n} \frac{A_{i}}{i !}
\end{aligned}
$$

so that

$$
A^{\prime}(t)=A(t)+t A^{\prime}(t)+\frac{A(t)}{1-t} .
$$

It follows that

$$
\frac{d}{d t} \ln (A(t))=\frac{A^{\prime}(t)}{A(t)}=\frac{1}{1-t}+\frac{1}{(1-t)^{2}} .
$$

Integrating and using the fact that $A(0)=1$, we see that

$$
\begin{aligned}
\ln (A(t)) & =-\ln (1-t)+\frac{1}{1-t}-1 \\
& =-\ln (1-t)+\frac{t}{1-t}
\end{aligned}
$$


which implies that

$$
A(t)=\frac{e^{t /(1-t)}}{1-t}
$$

This type of argument can be generalized. That is, we have the following theorem.

Theorem 7. Let $1 \leq r \leq s \leq k-1$ and $\vec{A}_{r, s, k}=$ $(\{0, \ldots, r-1\},\{s, \ldots, k-1\})$, where $k \geq 2$. Let $A(n, r, s, k)=$

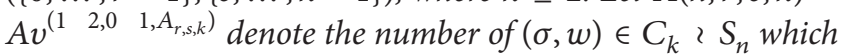
avoid (1 2, 0 1, $\left.\vec{A}_{r, s, k}\right)$, and

$$
A_{r, s, k}(t)=\sum_{n \geq 0} A(n, r, s, k) \frac{t^{n}}{n !}
$$

Then if $k-r \neq s$,

$$
A_{r, s, k}(t)=\frac{1}{1-(k-r) t}\left(\frac{1-s t}{1-(k-r) t}\right)^{r /(k-r-s)} .
$$

If $k-r=s$, then

$$
A_{r, k-r, k}(t)=\frac{e^{r t /(1-(k-r) t)}}{1-(k-r) t}
$$

Proof. Note that if $(\sigma, w) \in C_{k}<S_{n+1}$ contains the element 1 colored with a color from $\{r, \ldots, k-1\}$, then there are no restrictions for placing this element, thereby giving $(n+1) A(n, r, s, k)$ possibilities. On the other hand, if $(\sigma, w)$ contains the element 1 colored by the color $a$ in position $i+1$ where $a \in\{0, \ldots, r-1\}$, then if $(\sigma, w)$ is to avoid ( $12,01, \vec{A})$, it must be the case that every element to the right of 1 must be colored with a color from $\{0, \ldots, s-$ 1 ) and these elements can be arranged in any $(n-i)$ ! ways. Moreover, it immediately follows that no instance of a occurrence of ( 12 2, $\left.01, \vec{A}_{r, s, k}\right)$ exists where the first element is to the left of 1 and the second is to the right of 1 . Then it follows that $(\sigma, w)$ avoids $\left(\begin{array}{lll}1 & 2,0 & 1, \vec{A}_{r, s, k}\end{array}\right)$ if and only if there is no occurrence of $\left(\begin{array}{lll}1 & 2,0 & \left.1, \vec{A}_{r, s, k}\right)\end{array}\right)$ in $\left(\sigma_{1} \cdots \sigma_{i}, w_{1} \cdots w_{i}\right)$. Thus in the case where 1 is colored by a color from $\{0, \ldots, r-1\}$ and is in position $i+1$, we have $s^{n-i}(n-i) !\left(\begin{array}{c}n \\ i\end{array}\right) A(i, r, s, k)=n ! j^{n-i} A(i, r, s, k) / i !$ possibilities where the binomial coefficient is responsible for choosing the elements to the left of 1 and placing the remaining elements to the right of 1 . Hence

$$
\begin{aligned}
A(n+1, r, s, k)= & (k-r)(n+1) A(n, r, s, k) \\
& +r n ! \sum_{i=0}^{n} \frac{A(i, r, s, k)}{i !} s^{n-i} .
\end{aligned}
$$

Multiplying both parts of the equation above by $t^{n} / n !$ and summing over all $n \geq 0$, we have

$$
\begin{aligned}
\sum_{n \geq 0} A(n+1, r, s, k) \frac{t^{n}}{n !} & =(k-r) \sum_{n \geq 0} A(n, r, s, k) \frac{t^{n}}{n !} \\
& +(k-r) \sum_{n \geq 1} n A(n, r, s, k) \frac{t^{n}}{n !} \\
& +r \sum_{m \geq 0} t^{n} \sum_{i=0}^{n} \frac{A(i, r, s, k)}{i !} s^{n-i},
\end{aligned}
$$

so that for all $k \geq 2$,

$$
\begin{aligned}
A_{r, s, k}^{\prime}(t)= & (k-r) A_{r, s, k}(t) \\
& +(k-r) t A_{r, s, k}^{\prime}(t)+r \frac{A_{r, s, k}(t)}{1-s t} .
\end{aligned}
$$

It follows that

$$
\begin{aligned}
\frac{d}{d t} \ln \left(A_{r, s, k}(t)\right)= & \frac{A_{r, s, k}^{\prime}(t)}{A_{r, s, k}(t)}=\frac{k-r}{1-(k-r) t} \\
& +\frac{r}{(1-(k-r) t)(1-s t)}
\end{aligned}
$$

Now suppose that $k-r \neq s$. Then integrating (31) and using the fact that $A(0, r, s, k)=1$ and the fact that $(d / d t)(\ln (1-x t)-\ln (1-y t))=(y-x) /(1-x t)(1-y t)$, we see that

$$
\begin{aligned}
\ln \left(A_{r, s, k}(t)\right)= & -\ln (1-(k-r) t)+\frac{r}{(k-r-s)} \\
& \times(\ln (1-s t)-\ln (1-(k-r) t)),
\end{aligned}
$$

which implies that

$$
A_{r, s, k}(t)=\frac{1}{1-(k-r) t}\left(\frac{1-s t}{1-(k-r) t}\right)^{r /(k-r-s)}
$$

which proves (26).

Next suppose that $k-r=s$. Then (31) becomes

$$
\begin{aligned}
\frac{d}{d t} \ln \left(A_{r, k-r, k}(t)\right)= & \frac{A_{r, k-r, k}^{\prime}(t)}{A_{r, k-r, k}(t)} \\
& =\frac{j}{1-(k-r) t}+\frac{r}{(1-(k-r) t)^{2}} .
\end{aligned}
$$

Integrating (34) and using the fact that $A(0, r, k-r, k)=1$, we see that

$$
\begin{aligned}
\ln \left(A_{r, k-r, k}(t)\right)= & -\ln (1-(k-r) t) \\
& +\frac{r}{k-r}\left(\frac{1}{1-(k-r) t}-1\right) \\
= & -\ln (1-(k-r) t)+\frac{r t}{1-(k-r) t} .
\end{aligned}
$$


Thus

$$
A_{r, k-r, k}(t)=\frac{e^{r t /(1-(k-r) t)}}{1-(k-r) t}
$$

which proves (27).

Next consider the special case where $2 r=k$ and $r=s$ so $\vec{A}_{r, r, 2 r}=(\{0, \ldots, r-1\},\{r, \ldots, 2 r-2\})$. Then clearly,

$$
A_{r, r, 2 r}(t)=\frac{e^{r t /(1-r t)}}{1-r t}, \quad A_{1,1,2}(t)=\frac{e^{t /(1-t)}}{1-t} .
$$

It follows from the form of these generating functions that $A(n, r, r, 2 r)=r^{n} A(n, 1,1,2)$. Moreover, in the case where $k=2$, it is easy to see that our definitions imply that the number of $(\sigma, w) \in C_{2}, S_{n}$ which avoid ((1 2,01$\left.),(\{0\},\{1\})\right)$ is the same as the number of $(\sigma, w) \in C_{2}$ ? $S_{n}$ which avoid (1 2,0 1). Then $A(n, r, r, 2 r)=r^{n} A_{n}$ where $A_{n}$ is the number of $(\sigma, w) \in C_{2}, S_{n}$ avoiding (1 2,0 1). This is also easy to see combinatorially. Namely, if $(\sigma, w) \in C_{2 r} ? S_{n}$ which avoids $\left(\left(\begin{array}{lll}1 & 2,0 & 1\end{array}\right),(\{0, \ldots r-1\},\{r, \ldots, 2 r-1\})\right)$, then if $w^{\prime}$ is the result of replacing each occurrence of $0, \ldots, r-1$ in $w$ by 0 and each occurrence of $r, \ldots, 2 r-1$ in $w$ by 1 , then $\left(\sigma, w^{\prime}\right)$ will be an element of $C_{2}>S_{n}$ which avoids (1 2,0 1). Vice versa, if $\left(\sigma, w^{\prime}\right) \in C_{2}, S_{n}$ avoids $\left(\begin{array}{lll}1 & 2,0 & 1\end{array}\right)$ and $w$ arises from $w$ ' by replacing each 0 by some element from $\{0, \ldots, r-1\}$ and each 1 by some element from $\{r, \ldots, 2 j-1\}$, then $(\sigma, w)$ will avoid $((12,01),(\{0, \ldots, r-1\},\{r-1, \ldots, 2 r-1\}))$. In fact, if we are in the case where $r=s$ so that $\vec{A}_{r, r, k}=(\{0, \ldots, r-$ $1\},\{r-1, \ldots, k\})$, then by the same argument, we can get an $\operatorname{arbitrary}(\sigma, w) \in C_{k}, S_{n}$ which avoids $\left(\left(\begin{array}{lll}1 & 2,0 & 1\end{array}\right), \vec{A}_{r, r, k}\right)$ by starting out with an $\left(\sigma, w^{\prime}\right) \in C_{2} \geq S_{n}$ which avoids $\left(\begin{array}{lll}1 & 2,0 & 1\end{array}\right)$ and replacing each 0 by some element from $\{0, \ldots, r-1\}$ and each 1 by some element from $\{r-1, \ldots, k-1\}$. Considering the form of the generating function for $A_{r, r, k}(t)$ when $r \neq k$,

$$
A_{r, r, k}(t)=\frac{1}{1-(k-r) t}\left(\frac{1-r t}{1-(k-r) t}\right)^{r /(k-2 r)},
$$

this suggests the following theorem which can easily be proved by the same method as we used to prove Theorem 7 .

Theorem 8. Given $(\sigma, w) \in C_{2} \geq S_{n}$, let $\operatorname{pos}(\sigma, w)$ equal the number of 0 's in $w$ and neg $(\sigma, w)$ equal the number of 1 's in $w$. Let $\left.\mathbb{A}_{n}^{(1} \quad 2,01\right)$ denote the set of $(\sigma, w) \in C_{2}>S_{n}$ which avoids (1 2,0 1). Then

$$
\begin{aligned}
& A(t, y, z)=\sum_{n \geq 0} \frac{t^{n}}{n !} \sum_{\left.(\sigma, w) \in \mathbb{A}_{n}^{(1)} \quad 2,0 \quad 1\right)} y^{\operatorname{pos}(\sigma, w)} z^{n e g(\sigma, w)} \\
& =\frac{1}{1-z t}\left(\frac{1-y t}{1-z t}\right)^{y /(z-y)} .
\end{aligned}
$$

\section{Avoidance for Sets of Patterns}

In this section, we shall prove a variety of results for the number of elements of $C_{k}<S_{n}$ that avoid certain sets of patterns of length 2 .
If $\Upsilon \subseteq S_{j}$ is a set of permutations of $S_{j}$, we let

$$
\phi^{\Upsilon}(t)=\sum_{n \geq 0} A v_{n}^{\Upsilon} \frac{t^{n}}{n !},
$$

where $A v_{n}^{\Upsilon}$ is the number of permutations $\sigma \in S_{n}$ such that $\sigma$ avoids $\Upsilon$. If $\Upsilon \subseteq C_{k}>S_{j}$ and $\vec{A}=\left(A_{1}, A_{2}, \ldots, A_{j}\right)$ where $A_{i} \subseteq$ [k] for $i=1, \ldots, j$, then we let $A v_{n, k}^{(\Upsilon, \vec{A})}$ denote the number of $(\sigma, w) \in C_{k}$ ? $S_{n}$ which avoid $(\Upsilon, \vec{A})$. We then let

$$
\theta_{k}^{(\Upsilon, \vec{A})}(t)=\sum_{n \geq 0} A v_{n, k}^{(\Upsilon, \vec{A})} \frac{t^{n}}{n !}
$$

In the special case where for all $(\sigma, u) \in \Upsilon$, $\operatorname{red}(u)=u$ and $A_{i}=[k]$ for $i=1, \ldots, j$, we shall write $A v_{n, k}^{\Upsilon}$ instead of $A v_{n, k}^{(\Upsilon, \vec{A})}$. That is, $A v_{n, k}^{\Upsilon}$ is the number of $(\sigma, w) \in C_{k} 2 S_{n}$ which avoid $\Upsilon$. Then we let

$$
\theta_{k}^{\Upsilon}(t)=\sum_{n \geq 0} A v_{n, k}^{\Upsilon} \frac{t^{n}}{n !} .
$$

We start with a few simple results on sets of patterns $\Upsilon$ where the avoidance of $\Upsilon$ forces certain natural conditions on the possible sets of signs for elements of $C_{k}$ ? $S_{n}$.

Lemma 9. (1) If $\Upsilon_{1}=\left\{\left(\begin{array}{lll}1 & 2,0 & 0\end{array}\right),\left(\begin{array}{lll}2 & 1,0 & 0\end{array}\right)\right.$, then $A v_{n, k}^{\Upsilon_{1}}=$ $\left(\begin{array}{l}k \\ n\end{array}\right) n !^{2}$ for all $n \geq 1$ and $k \geq 1$.

(2) If $\Upsilon_{2}=\left\{\left(\begin{array}{lll}1 & 2,1 & 0\end{array}\right),\left(\begin{array}{lll}2 & 1,1 & 0\end{array}\right)\right.$, then $A v_{n, k}^{\Upsilon_{2}}=$ $\left(\begin{array}{c}n+k-1 \\ n\end{array}\right) n$ ! for all $n \geq 1$ and $k \geq 1$.

(3) If $\Upsilon_{3}=\left\{\left(\begin{array}{lll}1 & 2,0 & 0\end{array}\right),\left(\begin{array}{llll}1 & 2,1 & 0\end{array}\right),\left(\begin{array}{lll}2 & 1,0 & 0\end{array}\right)\right.$, (2 1,10$)\}$, then $A v_{n, k}^{Y_{3}}=\left(\begin{array}{l}k \\ n\end{array}\right) n$ ! for all $n \geq 1$ and $k \geq 1$.

(4) If $\Upsilon_{4}=\left\{\left(\begin{array}{lll}1 & 2,0 & 1\end{array}\right),\left(\begin{array}{lll}1 & 2,1 & 0\end{array}\right),\left(\begin{array}{llll}2 & 1,0 & 1\end{array}\right)\right.$, (2 1,10$)\}$, then $A v_{n, k}^{Y_{3}}=k n$ ! for all $n \geq 1$ and $k \geq 1$.

Proof. For (1), it is easy to see that $(\sigma, w) \in C_{k}<S_{n}$ avoids $\Upsilon_{1}$ if and only if all the signs are pairwise distinct. Therefore, there are $\left(\begin{array}{l}k \\ n\end{array}\right)$ ways to pick the $n$ signs, and then you have $n$ ! ways to arrange those $n$ signs and $n$ ! ways to pick $\sigma$.

For (2), it is easy to see that $(\sigma, w) \in C_{k}>S_{n}$ avoids $\Upsilon_{2}$ if and only if $0 \leq w_{1} \leq \cdots \leq w_{n} \leq k-1$. Therefore, there are $\left(\begin{array}{c}n+k-1 \\ n\end{array}\right)$ ways to pick the $n$ signs in this case and there are $n$ ! ways to pick $\sigma$.

For (3), it is easy to see that $(\sigma, w) \in C_{k}>S_{n}$ avoids $\Upsilon_{2}$ if and only if $0 \leq w_{1}<\cdots<w_{n} \leq k-1$. Therefore, there are $\left(\begin{array}{l}k \\ n\end{array}\right)$ ways to pick the $n$ signs in this case and there are $n$ ! ways to pick $\sigma$.

For (4), it is easy to see that $(\sigma, w) \in C_{k} \geq S_{n}$ avoids $\Upsilon_{2}$ if and only if $0 \leq w_{1}=\cdots=w_{n} \leq k-1$. Therefore, there are $k$ ways to pick the $n$ signs in this case and there are $n$ ! ways to pick $\sigma$.

For $\Upsilon \subset S-J$, we let

$$
\Upsilon_{0}=\left\{\left(\sigma, 0^{j}\right): \sigma \in \Upsilon\right\} .
$$

Then we have the following. 
Theorem 10. Let $\Upsilon \subseteq S_{j}$ be any set of permutations of $S_{j}$. Then if $\Gamma=\Upsilon_{0} \cup\left\{\left(\begin{array}{lll}1 & 2,1 & 0\end{array}\right),\left(\begin{array}{lll}2 & 1,1 & 0\end{array}\right)\right.$,

$$
\theta_{k}^{\Gamma}(t)=\left(\phi^{\Upsilon}(t)\right)^{k}
$$

for all $k \geq 1$.

Proof. It is easy to see that if $(\sigma, u) \in C_{k}>S_{n}$ and $(\sigma, u)$ avoids $\{(12,10),(21,10)\}$, then it must be the case that $0 \leq u_{1} \leq u_{2} \leq \cdots \leq u_{n} \leq k-1$. So suppose that $w=0^{a_{1}} 1^{a_{2}} \cdots(k-1)^{a_{k}}$ where $a_{1}+\cdots+a_{k}=n$. Then clearly the number of $\sigma \in S_{n}$ such that $(\sigma, w)$ avoids $\Upsilon_{0}$ is

$$
\left(\begin{array}{c}
n \\
a_{1}, \ldots, a_{k}
\end{array}\right) A v_{a_{1}}^{\Upsilon} \cdots A v_{a_{k}}^{\Upsilon} \text {. }
$$

That is, the binomial coefficient allows us to choose the elements $C_{i}$ of $\{1, \ldots, n\}$ that correspond to the constant segment $i^{a_{i+1}}$ in $w$. Then we only have to arrange the elements of $C_{i}$ so that it avoids $\Upsilon$ which can be done in $A v_{a_{i+1}}^{\Upsilon}$ ways. Thus it follows that

$$
A v_{n, k}^{\Gamma}=\sum_{\substack{a_{1}+\cdots+a_{k}=n \\
a_{i} \geq 0}}\left(\begin{array}{c}
n \\
a_{1}, \ldots, a_{k}
\end{array}\right) A v_{a_{1}}^{\Upsilon} \cdots A v_{a_{k}}^{\Upsilon},
$$

or, equivalently, that

$$
\frac{A v_{n, k}^{\Gamma}}{n !}=\sum_{\substack{a_{1}+\cdots+a_{k}=n \\ a_{i} \geq 0}} \prod_{i=1}^{k} \frac{A v_{a_{i}}^{\Upsilon}}{a_{i} !},
$$

which implies (44).

We immediately have the following corollary.

Corollary 11. For any $k \geq 1$, the number of elements of $C_{k}$ ? $S_{n}$ which avoid $\Gamma_{1}=\left\{\left(\begin{array}{lll}1 & 2,0 & 0\end{array}\right),\left(\begin{array}{llll}1 & 2,1 & 0\end{array}\right),\left(\begin{array}{lll}2 & 1,1 & 0\end{array}\right)\right\}$ or $\Gamma_{2}=\left\{(21,00),\left(\begin{array}{lll}1 & 2,1 & 0\end{array}\right),\left(\begin{array}{lll}2 & 1,1 & 0\end{array}\right)\right\}$ is $k^{n}$.

Proof. Let $\Upsilon_{1}=\left\{\begin{array}{ll}1 & 2\end{array}\right\}$ and $\Upsilon_{2}=\left\{\begin{array}{ll}2 & 1\end{array}\right\}$. Then clearly, $A v_{n}^{\Upsilon_{i}}=1$ for $i=1,2$ so that $\phi_{n}^{Y_{i}}(t)=e^{t}$ for $i=1,2$. But then by Theorem $10, \theta_{n, k}^{\Gamma_{i}}(t)=\left(e^{t}\right)^{k}=e^{k t}$ so that $A v_{n, k}^{\Gamma_{i}}=k^{n}$ for $i=1,2$.

We can derive theorems analogous to Theorem 10 for the other sign conditions in Lemma 9. That is, suppose that $\Upsilon$ is any set of patterns from $S_{j}$. Then we let

$$
\begin{gathered}
\Upsilon_{i}=\{(\tau, 0 \quad 1 \cdots(j-1)): \tau \in \Upsilon\}, \\
\Upsilon_{d}=\left\{(\tau, u): \tau \in \Upsilon, u \in D_{j}\right\},
\end{gathered}
$$

where $D_{j}$ is the set of all permutations of $\{0,1, \ldots, j-1\}$. Then we have the following.

Theorem 12. For any $\Upsilon \subset S_{j}$, let

$$
\begin{aligned}
& \Gamma_{1}=\Upsilon_{0} \cup\left\{\left(\begin{array}{lll}
1 & 2,0 & 1
\end{array}\right),\left(\begin{array}{lll}
1 & 2,1 & 0
\end{array}\right),\left(\begin{array}{lll}
2 & 1,0 & 1
\end{array}\right),\left(\begin{array}{lll}
2 & 1,1 & 0
\end{array}\right),\right. \\
& \Gamma_{2}=\Upsilon_{i} \cup\left\{\left(\begin{array}{llll}
1 & 2,1 & 0
\end{array}\right),\left(\begin{array}{llll}
1 & 2,0 & 0
\end{array}\right),\left(\begin{array}{lll}
2 & 1,1 & 0
\end{array}\right),\left(\begin{array}{lll}
2 & 1,0 & 0
\end{array}\right),\right. \\
& \Gamma_{3}=\Upsilon_{d} \cup\left\{\left(\begin{array}{llll}
1 & 2,0 & 0
\end{array}\right),\left(\begin{array}{lll}
2 & 1,0 & 0
\end{array}\right) .\right.
\end{aligned}
$$

Then, for all $k \geq 1$,
(1) $A v_{n, k}^{\Gamma_{1}}=k A v_{n}^{\Upsilon}$ for all $n \geq 1$,
(2) $A v_{n, k}^{\Gamma_{2}}=\left(\begin{array}{l}k \\ n\end{array}\right) A v_{n}^{\Upsilon}$ for all $n \geq 1$,
(3) $A v_{n, k}^{\Gamma_{3}}=\left(\begin{array}{l}k \\ n\end{array}\right) n ! A v_{n}^{\Upsilon}$ for all $n \geq 1$.

Proof. For (1), note that for $(\sigma, w) \in C_{k}<S_{n}$ to avoid $\{(12,01),(12,10),(21,01),(21,10)\}$, we must have $w=i^{n}$ for some $i \in\{0, \ldots, k-1\}$. Then $\left(\sigma, i^{n}\right) \in C_{k}>S_{n}$ avoids $\Upsilon_{0}$ if and only if $\sigma$ avoids $\Upsilon$. Thus $A v_{n, k}^{\Gamma_{1}}=k A v_{n}^{\Upsilon}$.

For (2), note that for $(\sigma, w) \in C_{k}<S_{n}$ to avoid $\left\{\left(\begin{array}{lll}1 & 2,1 & 0\end{array}\right),\left(\begin{array}{lll}1 & 2,0 & 0\end{array}\right),\left(\begin{array}{lll}2 & 1,1 & 0\end{array}\right),\left(\begin{array}{lll}2 & 1,0 & 0\end{array}\right)\right\}$, we must have $w=w_{1} \cdots w_{n}$ where $w_{1}<\cdots<w_{n}$. Then for any of the $\left(\begin{array}{l}k \\ n\end{array}\right)$ strictly increasing words $w \in\{0, \ldots, k-1\}$, $(\sigma, w) \in C_{k}, S_{n}$ avoids $\Upsilon_{i}$ if and only if $\sigma$ avoids $\Upsilon$. Thus $A v_{n, k}^{\Gamma_{2}}=\left(\begin{array}{l}k \\ n\end{array}\right) A v_{n}^{\Upsilon}$.

For (3), note that, for $(\sigma, w) \in C_{k} \geq S_{n}$ to avoid $\left\{\left(\begin{array}{lll}1 & 2,0 & 0\end{array}\right),\left(\begin{array}{lll}2 & 1,0 & 0\end{array}\right)\right\}$, we must have $w=w_{1} \cdots w_{n}$ where the letters of $w$ are pairwise distinct. Then for any of the $\left(\begin{array}{l}k \\ n\end{array}\right) n$ ! words $w \in\{0, \ldots, k-1\}$ which have pairwise distinct letters, $(\sigma, w) \in C_{k}, S_{n}$ avoids $\Upsilon_{d}$ if and only if $\sigma$ avoids $\Upsilon$. Thus $A v_{n, k}^{\Gamma_{3}}=\left(\begin{array}{l}k \\ n\end{array}\right) n ! A v_{n}^{\Upsilon}$.

Next we will prove two more results about $A v_{n, k}^{\Upsilon}$ for other sets of patterns $\Upsilon$ that contain $\left\{\left(\begin{array}{llll}1 & 2,1 & 0\end{array}\right),\left(\begin{array}{lll}2 & 1,1 & 0\end{array}\right)\right.$.

Theorem 13. Let

$$
\begin{gathered}
\Upsilon_{1}=\left\{\left(\begin{array}{lll}
1 & 2,0 & 1
\end{array}\right),\left(\begin{array}{llll}
1 & 2,1 & 0
\end{array}\right),\left(\begin{array}{lll}
2 & 1,1 & 0
\end{array}\right),\right. \\
\Upsilon_{2}=\left\{\left(\begin{array}{lllll}
1 & 2,0 & 1
\end{array}\right),\left(\begin{array}{lllll}
1 & 2,1 & 0
\end{array}\right),\left(\begin{array}{llll}
2 & 1,1 & 0
\end{array}\right),\left(\begin{array}{lll}
2 & 1,0 & 0
\end{array}\right) .\right.
\end{gathered}
$$

Then

(1) $A v_{n, k}^{\Upsilon_{1}}=\sum_{\substack{a_{i} \geq 0 \\ a_{1}+\cdots+a_{k}=n}} a_{1} ! \cdots a_{k}$ ! for all $n \geq 1$ and $k \geq 1$,

(2) $A v_{n, k}^{\Upsilon_{1}}=\left(\begin{array}{c}n+k-1 \\ k-1\end{array}\right)$ for all $n \geq 1$ and $k \geq 1$.

Proof. For (1), note that as in the proof of Theorem 10, if $(\sigma, u) \in C_{k} 2 S_{n}$ and $(\sigma, u)$ avoids $\left\{\left(\begin{array}{llll}1 & 2,1 & 0\end{array}\right),\left(\begin{array}{lll}2 & 1,1 & 0\end{array}\right)\right.$, then it must be the case that $0 \leq u_{1} \leq u_{2} \leq \cdots \leq u_{n} \leq k-1$.

Now suppose that $w=0^{a_{1}} 1^{a_{2}} \cdots(k-1)^{a_{k}}$ where $a_{1}+$ $\cdots+a_{k}=n$. Assume that $(\sigma, w)$ also avoids ( 12,01$)$ and $C_{i}$ is the set of elements of $\sigma$ that correspond to the signs $(i-1)^{a_{i}}$ in $w$. Then it follows that all the elements of $C_{1}$ must be larger than all the elements of $C_{2}$, all the elements of $C_{2}$ must be larger than all the elements of $C_{3}$, and so forth. Thus $C_{1}$ consists of the $a_{1}$ largest elements of $\{1, \ldots, n\}, C_{2}$ consists of next $a_{2}$ largest elements of $\{1, \ldots, n\}$, and so forth. Then we can arrange the elements of $C_{i}$ in any order in the positions corresponding to $(i-1)^{a_{i}}$ in $w$ to produce a $(\sigma, w)$ which avoids $\Upsilon$. Hence there are $a_{1} ! \cdots a_{k}$ ! elements of the form $(\sigma, w)$ which avoid $\Upsilon$ in $C_{k}>S_{n}$. Therefore (1) immediately follows.

For (2), observe that if in addition such $(\sigma, w)$ also avoids (2 $1,0 \quad 0)$, then we must place the elements of $C_{i}$ in increasing order. Hence $A v_{n, k}^{Y_{2}}$ is the number of solutions of $a_{1}+\cdots+a_{k}=n$ with $a_{i} \geq 0$ which is well known to be $\left(\begin{array}{c}n+k-1 \\ k-1\end{array}\right)$. 
Theorem 14. Let $\Upsilon=\left\{\left(\begin{array}{lll}1 & 2,0 & 1\end{array}\right),\left(\begin{array}{llll}1 & 2,1 & 0\end{array}\right),\left(\begin{array}{lll}2 & 1,0 & 0\end{array}\right)\right.$. Then for $n \geq 1$,

$$
\begin{gathered}
A v_{n, 1}^{\Upsilon}=1, \\
A v_{n, 2}^{\Upsilon}=2 n, \\
A v_{n, k}^{\Upsilon}=k+\sum_{j=2}^{k-1}(k) \downarrow_{j}\left(\begin{array}{l}
n \\
j
\end{array}\right) \quad \text { for } k \geq 3,
\end{gathered}
$$

where $(k) \downarrow_{0}=1$ and $(k) \downarrow_{n}=k(k-1) \cdots(k-n+1)$ for $n \geq 1$.

Proof. We shall classify the elements $(\sigma, w) \in C_{k}>S_{n}$ which avoid $\Upsilon$ by the number of elements $s$ which follow $n$ in $\sigma$. Now if $s=0$ so that $\sigma$ ends in $n$, the fact that $(\sigma, w)$ avoids both ( $\left.\begin{array}{lll}1 & 2,0 & 1\end{array}\right)$ and (1 $\left.2,1 \quad 0\right)$ means that all the signs must be the same. That is, $w$ must be of the form $i^{n}$ for some $i \in[k]$. But since $(\sigma, w)$ must also avoid ( $2 \quad 1,0 \quad 0), \sigma=1 \quad 2 \quad \ldots \quad n$ must be the identity. Thus there are $k$ choices for such $(\sigma, w)$.

Now suppose there are $s$ elements following $n$ in $\sigma$. Then again the fact that $(\sigma, w)$ avoids both $\left(\begin{array}{lll}1 & 2,0 & 1\end{array}\right)$ and $\left(\begin{array}{lll}1 & 2,1 & 0\end{array}\right)$ means that all the signs in $w$ corresponding to $\sigma_{1} \cdots \sigma_{n-s}\left(\right.$ where $\sigma_{n-s}=n$ ) must be the same, say that sign is $i$. The fact that $(\sigma, w)$ avoids $(2 \quad 1,00)$ means that (i) $\sigma_{1} \cdots \sigma_{n-s}$ must be in increasing order and (ii) all the signs corresponding to $\sigma_{n-s+1} \cdots \sigma_{n}$ must be different from $i$. But then it follows from the fact that $(\sigma, w)$ avoids both $\left(\begin{array}{lll}1 & 2,0 & 1\end{array}\right)$ and $\left(\begin{array}{lll}1 & 2,1 & 0\end{array}\right)$ that all the elements in $\sigma_{1} \cdots \sigma_{n-s}$ must be greater than all the elements in $\sigma_{n-s+1} \cdots \sigma_{n}$. Hence there are $k A v_{s, k-1}^{\gamma}$ such elements if $k \geq 2$ and there are no such elements if $k=1$. Thus from (ii) it follows that $A v_{n, 1}^{Y}=1$ since (1 $2 \cdots n, 0^{n}$ ) is the only element of $C_{k}>S_{n}$ which avoids $\Upsilon$. For $k \geq 2$, we have

$$
A v_{n, k}^{\Upsilon}=k+\sum_{s=1}^{n-1} k A v_{s, k-1}^{\Upsilon} .
$$

In the case $k=2$, (52) becomes

$$
A v_{n, 2}^{\Upsilon}=2+\sum_{s=1}^{n-1} 2=2 n
$$

and in the case $k=3$, (52) becomes

$$
A v_{n, 3}^{\Upsilon}=3+\sum_{s=1}^{n-1} 3(2 s)=3+(3 \cdot 2)\left(\begin{array}{l}
n \\
2
\end{array}\right) .
$$

In general, assuming that

$$
A v_{n, k}^{\Upsilon}=k+\sum_{j=2}^{k-1}(k) \downarrow_{j}\left(\begin{array}{c}
n \\
j
\end{array}\right)
$$

it follows that

$$
\begin{aligned}
A v_{n, k+1}^{\Upsilon}= & k+1+\sum_{s=1}^{n-1}(k+1) A v_{s, k}^{\Upsilon} \\
= & k+1+\sum_{s=1}^{n-1}(k+1)\left(k+\sum_{j=2}^{k-1}(k) \downarrow_{j}\left(\begin{array}{l}
s \\
j
\end{array}\right)\right) \\
= & k+1+\left(\sum_{s=1}^{n-1}(k+1) k\right) \\
& +\sum_{j=2}^{k-1}(k+1)(k) \downarrow_{j} \sum_{s=1}^{n-1}\left(\begin{array}{l}
s \\
j
\end{array}\right) \\
= & k+1+(k+1) \downarrow_{2}\left(\begin{array}{l}
n \\
2
\end{array}\right) \\
& +\sum_{j=3}^{k}(k+1) \downarrow_{j}\left(\begin{array}{l}
n \\
j
\end{array}\right) .
\end{aligned}
$$

We next consider simultaneous avoidance of the patterns $\left(\begin{array}{lll}1 & 2,1 & 0\end{array}\right)$ and (1 2,0 1).

Theorem 15. Let $\Upsilon=\left\{\left(\begin{array}{lll}1 & 2,1 & 0\end{array}\right),\left(\begin{array}{lll}1 & 2,0 & 1\end{array}\right), A_{k}^{\Upsilon}(t)=\right.$ $\sum_{n \geq 0} A v_{n, k}^{Y} t^{n}$ and $C(t)=\sum_{n \geq 1} n ! t^{n}$. Then

$$
A_{k}^{\curlyvee}(t)=\frac{1+C(t)}{1-(k-1) C(t)} .
$$

Proof. Fix $k$ and suppose that $(\sigma, w)$ is an element of $C_{k}>S_{n}$ which avoids both (1 $2,1 \quad 0)$ and $\left(\begin{array}{lll}1 & 2,0 & 1\end{array}\right)$. Now if $w=i^{n}$ is constant, then clearly $\sigma$ can be arbitrary so that there are $n$ ! such elements.

Next assume that $w$ is not constant so there is an $s \geq 2$ such that $w=i_{1}^{a_{1}} i_{2}^{a_{2}} \cdots i_{s}^{a_{s}}$ where $a_{r} \geq 1$ for $r=1, \ldots, s$ and $i_{r} \neq i_{r+1}$ for $r=1, \ldots, s-1$. We claim that $\sigma_{1} \cdots \sigma_{a_{1}}$ must be the $a_{1}$ largest elements of $\{1, \ldots, n\}$. If not, then let $n-r$ be the largest element which is not in $\sigma_{1} \cdots \sigma_{a_{1}}$. Thus $r<a_{1}-1$ and there must be at least one $\sigma_{t}$ with $t \leq s$ such that $\sigma_{t}<n-r$. Now it cannot be $\sigma_{a_{1}+1}=n-r$ since otherwise $\left(\sigma_{t} \sigma_{a_{1}+1}, i_{1} i_{2}\right)$ would be an occurrence of either (1 2, 10$)$ or $\left(\begin{array}{lll}1 & 2,0 & 1\end{array}\right)$. Hence it must be the case that $\sigma_{a_{1}+1}<n-r$ and $\sigma_{p}=n-r$ for some $p>a_{1}+1$. But then no matter what color we choose for $w_{p}$, either $\left(\sigma_{t} \sigma_{p}, i_{1} w_{p}\right)$ or $\left(\sigma_{a_{1}+1} \sigma_{p}, i_{2} \quad w_{p}\right)$ would be an occurrence of either $\left(\begin{array}{lll}1 & 2,1 & 0\end{array}\right)$ or (1 2, 01 ). We can continue this reasoning to show that for any $1 \leq p<q \leq s$, the elements of $\sigma$ corresponding to the block $i_{p}^{a_{p}}$ in $w$ must be strictly larger than the elements of $\sigma$ corresponding to the block $i_{q}^{a_{q}}$ in $w$. This given, it follows that we can arrange the elements of $\sigma$ corresponding to a block $i_{p}^{a_{p}}$ in $w$ in any way that we want and we will always produce a pair $(\sigma, w)$ that avoids both $\left(\begin{array}{llll}1 & 2,1 & 0\end{array}\right)$ and (1 2,0 1). Thus for such a $w$, we have $k(k-1)^{s-1}$ ways to choose the colors 
$i_{1}, \ldots, i_{s}$ and $a_{1} ! a_{2} ! \cdots a_{k}$ ! ways to choose the permutation $\sigma$. It follows that

$$
\begin{aligned}
A v_{n, k}^{\Upsilon}= & k n !+\sum_{s=2}^{n-1} \\
& \times \sum_{\substack{a_{1}+\cdots+a_{s}=n \\
a_{i}>0}} k(k-1)^{s-1} a_{1} ! a_{2} ! \cdots a_{s} !,
\end{aligned}
$$

which is equivalent to (57).

Next we want to consider the problem of avoiding $(\Upsilon, \vec{B})$ where $\Upsilon=\left\{\left(\begin{array}{lll}1 & 2,0 & 0\end{array}\right),\left(\begin{array}{lll}1 & 2,0 & 1\end{array}\right)\right\}$ and $\vec{B}=([k],\{k-1\})$. If $\sigma=\sigma_{1} \cdots \sigma_{n} \in S_{n}$, then we say that $\sigma_{j}$ is a left-to-right minimum of $\sigma$ if $\sigma_{i}>\sigma_{j}$ for all $i<j$ and we let $\operatorname{lrmin}(\sigma)$ denote the number of left-to-right minima of $\sigma$. Let $\mathbb{B}_{n, k}$ denote the set of all $(\sigma, w) \in C_{k} 2 S_{n}$ which avoid $(\Upsilon, \vec{B})$. Then we let

$$
B_{k}(x, t)=\sum_{n \geq 0} \frac{t^{n}}{n !} \sum_{(\sigma, w) \in \mathbb{B}_{n, k}} x^{\operatorname{lrmin}(\sigma)} .
$$

Then we have the following theorem.

Theorem 16. For $\Upsilon=\left\{\left(\begin{array}{lll}1 & 2,0 & 0\end{array}\right),\left(\begin{array}{lll}1 & 2,0 & 1\end{array}\right)\right\}$ and $\vec{B}=$ $([k],\{k-1\})$, one has

$$
B_{k}(x, t)=\left(\frac{1}{1-(k-1) t}\right)^{k x /(k-1)} .
$$

Proof. Let $B_{n, k}(x)=\sum_{(\sigma, w) \in \mathbb{B}_{n, k}} x^{\operatorname{lrmin}(\sigma)}$. If $(\sigma, w)$ contains the element 1 colored by the color $a$ in position $i+1$ where $a \in$ $\{0, \ldots, k-1\}$, then if $(\sigma, w)$ is to avoid $(\Upsilon, \vec{B})$, it must be the case that every element to the right of 1 must be colored with a color from $\{0, \ldots, k-2\}$ and these elements can be arranged in any of $(n-i)$ ! ways. Moreover, it immediately follows that no instance of an occurrence of $(\Upsilon, \vec{B})$ exists where the first element is to the left of 1 and the second is to the right of 1 . Thus it follows that $(\sigma, w)$ avoids $(\Upsilon, \vec{B})$ if and only if there is no occurrence of $(\Upsilon, \vec{B})$ in $\left(\sigma_{1} \cdots \sigma_{i}, w_{1} \cdots w_{i}\right)$. Thus it follows that

$$
\begin{aligned}
\sum_{(\sigma, w) \in \mathbb{B}_{n, k}, \sigma_{i+1}=1} x^{\operatorname{lrmin}(\sigma)}= & x(k-1)^{n-i} \\
& \times(n-i) !\left(\begin{array}{c}
n \\
i
\end{array}\right) B_{i, k}(x) \\
= & \frac{n ! x(k-1)^{n-i} B_{i, k}(x)}{i !} .
\end{aligned}
$$

Here the binomial coefficient is responsible for choosing the elements to the left of 1 and placing the remaining elements to the right of 1 and the factor of $x$ accounts for the fact that 1 is always a left-to-right minimum in $\sigma$ and none of the elements following 1 can be a left-to-right minimum of $\sigma$. This shows that

$$
B_{n+1, k}(x)=k x n ! \sum_{i=0}^{n} \frac{B_{i, k}(x)}{i !}(k-1)^{n-i} .
$$

Multiplying both parts of the equation above by $t^{n} / n$ ! and summing over all $n \geq 1$, we have

$$
\sum_{n \geq 0} B_{n+1, k}(x) \frac{t^{n}}{n !}=k x \sum_{m \geq 0} t^{n} \sum_{i=0}^{n} \frac{B_{i, k}(x)}{i !}(k-1)^{n-i},
$$

so that, for all $k \geq 2$,

$$
\frac{\partial}{\partial t} B_{k}(t, x)=k x \frac{B_{k}(t, x)}{1-(k-1) t} .
$$

It follows that

$$
\frac{\partial}{\partial t} \ln \left(B_{k}(x, t)\right)=\frac{k x}{1-(k-1) t} .
$$

Integrating and using the fact that $B_{k}(x, 0)=1$, we see that

$$
\left(B_{k}(x, t)\right)=\frac{-k x}{k-1} \ln (1-(k-1) t),
$$

and hence,

$$
B_{k}(x, t)=\left(\frac{1}{1-(k-1) t}\right)^{k x /(k-1)} .
$$

Using the fact that

$$
\frac{1}{(1-z)^{a}}=\sum_{n \geq 0} a(a+1) \cdots(a+n-1) \frac{z^{n}}{n !},
$$

we see that

$$
\begin{aligned}
B_{n, k}(x)= & \left(\frac{k x}{k-1}\right)\left(\frac{k x}{k-1}+1\right) \\
& \cdots\left(\frac{k x}{k-1}+n-1\right)(k-1)^{n} \\
= & (k x)(k x+(k-1)) \\
& \cdots(k x+(n-1)(k-1)) .
\end{aligned}
$$

In particular,

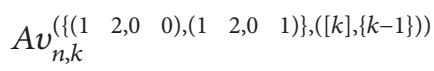

$$
\begin{aligned}
& =k(2 k-1)(3 k-2) \cdots(n k-(n-1)) .
\end{aligned}
$$

We note that, by our symmetry maps where we apply the identity map to $\sigma$ and the composition of complement and reverse to $w$ and $\vec{A}$, one can show that

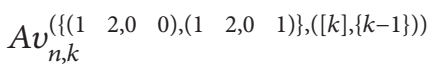

$$
\begin{aligned}
& \left.\left.\left.=A v_{n, k}^{(\{1} \quad 2, k-1 \quad k-1\right),(1 \quad 2, k-2 \quad k-1)\right\},(\{0\},[k])\right) .
\end{aligned}
$$

It is easy to see that our definitions imply that

$$
\begin{aligned}
& \left.\left.\left.A v_{n, k}^{(\{(1} \quad 2,0 \quad 0\right),(1 \quad 2,0 \quad 1)\right\},(\{0\},[k])\right)
\end{aligned}
$$

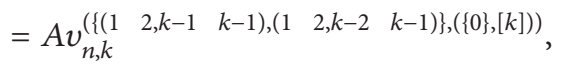


so that

$$
\begin{aligned}
& \left.\left.\left.A v_{n, k}^{(\{(1)} \begin{array}{llll}
2,0 & 0),(1 & 2,0 & 1
\end{array}\right)\right\},\{\{0\},[k])\right) \\
& \quad=k(2 k-1)(3 k-2) \cdots(n k-(n-1)) .
\end{aligned}
$$

Next we consider the case where $1<j<k$, $\vec{C}_{j}=(\{0, \ldots, j-1\},\{j-1, \ldots, k-1\})$, and $\Upsilon=$ $\left\{\left(\begin{array}{lll}1 & 2,0 & 0\end{array}\right),\left(\begin{array}{lll}1 & 2,0 & 1\end{array}\right)\right\}$.

Theorem 17. Let $\vec{C}_{j}=(\{0, \ldots, j-1\},\{j-1, \ldots, k-1\})$ where $k \geq 3$ and $1<j<k$, and $\Upsilon=\left\{\left(\begin{array}{lll}1 & 2,0 & 0\end{array}\right),\left(\begin{array}{lll}1 & 2,0 & 1\end{array}\right)\right\}$. Then if $k-j \neq j-1$,

$$
\theta_{k}^{\left(\Upsilon, \vec{C}_{j}\right)}(t)=\frac{1}{1-(k-j) t}\left(\frac{1-(j-1) t}{1-(k-j) t}\right)^{j /(k-2 j+1)}
$$

If $k-j=j-1$, then $k=2 j-1$ and

$$
\theta_{2 j-1}^{\left(\Upsilon, \vec{C}_{j}\right)}(t)=\frac{e^{j t /(1-(j-1) t)}}{1-(j-1) t}
$$

Proof. Let $C(n, k, j)=A v_{n, k}^{\left(\Upsilon, \vec{C}_{j}\right)}$ so that

$$
C_{k, j}(t)=\sum_{n \geq 0} C(n, k, j) \frac{t^{n}}{n !}=\theta_{k}^{\left(\Upsilon, \vec{C}_{j}\right)}(t) .
$$

Now suppose that $(\sigma, w) \in C_{k} 2 S_{n}$ contains the element 1 colored by a color from $\{j, \ldots, k-1\}$. Then there are no restrictions for placing this element, thus giving $(n+1)$ possibilities. On the other hand, if $(\sigma, w)$ contains the element 1 colored by the color $a$ in position $i+1$ where $a \in\{0, \ldots, j-1\}$, then if $(\sigma, w)$ is to avoid $\left(\Upsilon, \vec{C}_{j}\right)$, it must be the case that every element to the right of 1 must be colored with a color from $\{0, \ldots, j-2\}$ and these elements can be arranged in any of $(n-i)$ ! ways. Moreover, it immediately follows that no instance of an occurrence of $\left(\Upsilon, \vec{C}_{j}\right)$ exists where the first element is to the left of 1 and the second is to the right of 1 . Then it follows that $(\sigma, w)$ avoids $\left(\Upsilon, \vec{C}_{j}\right)$ if and only if there is no occurrence of $\left(\Upsilon, \vec{C}_{j}\right)$ in $\left(\sigma_{1} \cdots \sigma_{i}, w_{1} \cdots w_{i}\right)$. So in the case where 1 is colored by a color from $\{0, \ldots, j-1\}$ and is in position $i+1$, we have $(j-1)^{n-i}(n-i) !\left(\begin{array}{c}n \\ i\end{array}\right) C(i, k, j)=n !(j-$ $1)^{n-i} C(i, k, j) / i$ ! possibilities where the binomial coefficient is responsible for choosing the elements to the left of 1 and placing the remaining elements to the right of 1 . Thus

$$
\begin{aligned}
C(n+1, k, j)= & (k-j)(n+1) C(n, k, j) \\
& +j n ! \sum_{i=0}^{n} \frac{C(i, k, j)}{i !}(j-1)^{n-i} .
\end{aligned}
$$

Multiplying both parts of the equation above by $t^{n} / n$ ! and summing over all $n \geq 1$, we have

$$
\begin{aligned}
\sum_{n \geq 0} C(n+1, k, j) \frac{t^{n}}{n !}= & (k-j) \sum_{n \geq 0} C(n, k, j) \frac{t^{n}}{n !} \\
& +(k-j) \sum_{n \geq 1} n C(n, k, j) \frac{t^{n}}{n !} \\
& +j \sum_{m \geq 0} t^{n} \sum_{i=0}^{n} \frac{C(i, k, j)}{i !}(j-1)^{n-i},
\end{aligned}
$$

so that, for all $k \geq 3$ and $1<j<k$,

$$
\begin{aligned}
C_{k, j}^{\prime}(t)= & (k-j) C_{k, j}(t) \\
& +(k-j) t C_{k, j}^{\prime}(t)+j \frac{C_{k, j}(t)}{1-(j-1) t} .
\end{aligned}
$$

It follows that

$$
\begin{aligned}
\frac{d}{d t} \ln \left(C_{k, j}(t)\right)= & \frac{C_{k, j}^{\prime}(t)}{C_{k, j}(t)}=\frac{1}{1-(k-j) t} \\
& +\frac{j}{(1-(k-j) t)(1-(j-1) t)}
\end{aligned}
$$

Now suppose that $k-j \neq j-1$. Then integrating (80) and using the fact that $C(0, k, j)=1$ and that $(d / d t)(\ln (1-x t)-$ $\ln (1-y t))=(y-x) /(1-x t)(1-y t)$, we see that

$$
\begin{aligned}
\ln \left(C_{k, j}(t)\right)= & -\ln (1-(k-j) t) \\
& +\frac{j}{(k-j)-(j-1)} \\
& \times(\ln (1-j t)-\ln (1-(k-j) t))
\end{aligned}
$$

which implies that

$$
C_{k, j}(t)=\frac{1}{1-(k-j) t}\left(\frac{1-(j-1) t}{1-(k-j) t}\right)^{j /(k-2 j+1)}
$$

and proves (74).

Now if $k-j=j-1$. Then $k=2 j-1$ and (80) becomes

$$
\begin{aligned}
\frac{d}{d t} \ln \left(C_{2 j-1, j}(t)\right)= & \frac{C_{2 j-1, j}^{\prime}(t)}{C_{2 j-1, j}(t)}=\frac{j-1}{1-(j-1) t} \\
& +\frac{j}{(1-(j-1) t)^{2}} .
\end{aligned}
$$


Integrating (83) and using the fact that $C(0,2 j-1, j)=1$, we see that

$$
\begin{aligned}
\ln \left(C_{2 j-1, j}(t)\right)= & -\ln (1-(j-1) t) \\
& +\frac{j}{j-1}\left(\frac{1}{1-(j-1) t}-1\right) \\
= & -\ln (1-(j-1) t) \\
& +\frac{j t}{1-(j-1) t} .
\end{aligned}
$$

Thus

$$
C_{2 j-1, j}(t)=\frac{e^{j t /(1-(j-1) t)}}{1-(j-1) t},
$$

which proves (75).

Finally, we end this section by considering the elements of $C_{k}<S_{n}$ which avoid both (1 $\left.2,0 \quad 0\right)$ and ( $\left.12,0 \quad 1\right)$. In this case, we only have a result for the case when $k=2$.

Theorem 18. The number of permutations in $C_{2}>S_{n}$ simultaneously avoiding (1 2,0 0 ) and (1 2,0 1) is given by the $(n+1)$-th Catalan number $C_{n+1}=(1 /(n+2))\left(\begin{array}{c}2 n+2 \\ n+1\end{array}\right)$.

Proof. We prove the statement by establishing a bijection between the objects in question of length $n$ and the Dyck paths of semilength $(n+1)$ known to be counted by the Catalan numbers (a Dyck path of semi-length $n$ is a lattice path from $(0,0)$ to $(2 n, 0)$ with steps $(1,1)$ and $(1,-1)$ that never goes below the $x$-axis; Figure 4$)$.

Suppose $(\sigma, w) \in C_{2}>S_{n}$. Note that $\sigma$ must avoid the pattern 123 , as in an occurrence of such a pattern in $\sigma$, there are two letters of the same color leading to an occurrence of ( $\left(\begin{array}{lll}1 & 2,0\end{array}\right)$. Thus the structure of $\sigma$, as it is well known, is two decreasing sequences shuffled.

Subdivide $\sigma$ into the so-called reverse irreducible components. A reverse irreducible component is a factor $F$ of $\sigma$ of minimal length such that everything to the left (resp., right) of $F$ is greater (resp., smaller) than any element of $F$. For example, the subdivision of $\sigma=6574312$ is $\sigma=657-4-3-12$. The blocks of size 1 are called singletons. In the example above, 4 and 3 are singletons. It is easy to see that any singleton element in $\sigma$ can have any color (either 0 or 1 ). We will now show that the color of each element of a nonsingleton block is uniquely determined.

Indeed, irreducibility of a single block means that two decreasing sequences in the structure of $\left(\begin{array}{lll}1 & 2 & 3\end{array}\right)$-avoiding permutations are the block's sequence of left-to-right minima and the block's sequence of right-to-left maxima which do not overlap. Thus for any left-to-right minimal element $x$ (except possibly the last element), one has an element $y$ greater than $x$ to the right of it inside the same block and vice versa, from which we conclude that $x$ must receive color 1 , whereas $y$ must receive color 0 (otherwise a prohibited pattern will occur).

We are ready to describe our bijection. For a given $(\sigma, w) \in C_{2} 2 S_{n}$, consider a matrix representation of $\sigma$, that is, an integer grid with the opposite corners in $(0,0)$ and $(n, n)$ and with a dot in position $\left(i-1 / 2, \sigma_{i}-(1 / 2)\right)$ for $i=1,2, \ldots, n$. We will give a description of a path $P$ (corresponding to $(\sigma, w))$ from $(0, n+1)$ to $(n+1,0)$ involving only steps $(0,-1)$ and $(1,0)$ that never goes above the line $y=-x+n+1$. Clearly, $P$ can be transformed to a Dyck path of length $(n+1)$ by taking a mirror image with respect to the line $y=-x+n+1$, rotating 45 degrees counterclockwise and making a parallel shift.

To build $P$, set $i:=0$ and $j:=n+1$, and do the following steps, letting $P$ begin at $(i, j)$. Clearly, each reverse irreducible block of $\sigma$ defines a square on the grid which is a matrix representation of the block. We call the reverse irreducible block of $\sigma$ with the left-top corner at $(i, j)$ the current block.

Step 1. If the current block is not a singleton, go to Step 2. If the color of the element with $x$-coordinate equal to $i+1 / 2$ is 0 (resp., 1) travel around the current block counterclockwise (resp., clockwise) to get to the point $(i+1, j-1)$. Note that $P$ touches the line $y=-x+n+1$ if the color is 1 . Set $i:=i+1$ and $j:=j-1$, and proceed with Step 3.

Step 2. In Step 2 we follow a standard bijection between (1 $\left.2 \begin{array}{ll}1 & 3\end{array}\right)$-avoiding permutations and Dyck paths that can be described as follows. Let $(k, \ell)$ be the point of the current block opposite to $(i, j)$. Start going down from $(i, j)$ until the $y$-coordinate of the current node gets $1 / 2$ less than the $y$ coordinate of the dot with $x$-coordinate equal $i+1 / 2$. Start moving horizontally to the right and go as long as possible making sure that none of the dots are below the part of $P$ constructed so far and $i \leq k$ and $j \leq \ell$. Suppose $\left(i_{1}, j_{1}\right)$ is the last point the procedure above can be done (i.e., we were traveling on the line $y=j_{1}$ and either $i_{1}=k$ and $j_{1}=\ell$ or there is a dot with $x$-coordinate $i_{1}+(1 / 2)$ having $y$-coordinate less than $j_{1}$ ). If $i:=k$ and $j:=\ell$, proceed with Step 3; otherwise set $i:=i_{1}$ and $j:=j_{1}$ and go to Step 2 . Note that in Step $2 P$ never touches the line $y=-x+n+1$.

Step 3. If $j=0$, make as many as it takes horizontal steps to get to the point $(n+1,0)$ and terminate; otherwise go to Step 1.

Returning to our example, $\sigma=\left(\begin{array}{lllllll}6 & 5 & 7 & 4 & 3 & 1 & 2\end{array}\right.$, $\left.\begin{array}{lllllll}1 & 1 & 0 & 1 & 0 & 1 & 0\end{array}\right)$, we have given the matrix diagram in Figure 1. We have outlined the reverse irreducible blocks in Figure 2. We start our path at $(0,8)$. We travel down until we reach $(0,6)$, when we are $1 / 2$ less than the $y$ coordinate of our first point $(1 / 2,6(1 / 2))$. We then continue traveling right and down as described in Step 2. We travel clockwise around our singleton colored 1 and counterclockwise around our singleton colored 0 . Then we continue to the final reverse irreducible block and finish our path, given in Figure 3.

\section{Pattern Matching Results for Pairs of Length $\geq 2$}

Goulden and Jackson [8] proved the following theorem. 


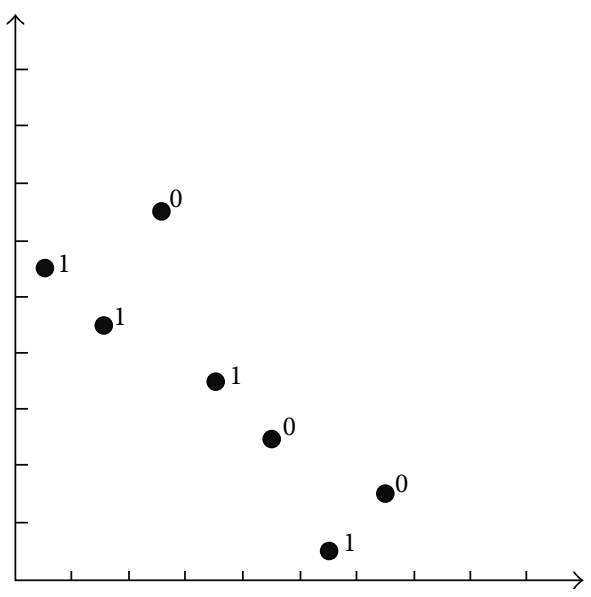

FIGURE 1: Matrix representation of $\sigma=\left(\begin{array}{lllllll}6 & 5 & 7 & 4 & 3 & 1 & 2\end{array}\right.$,

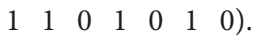

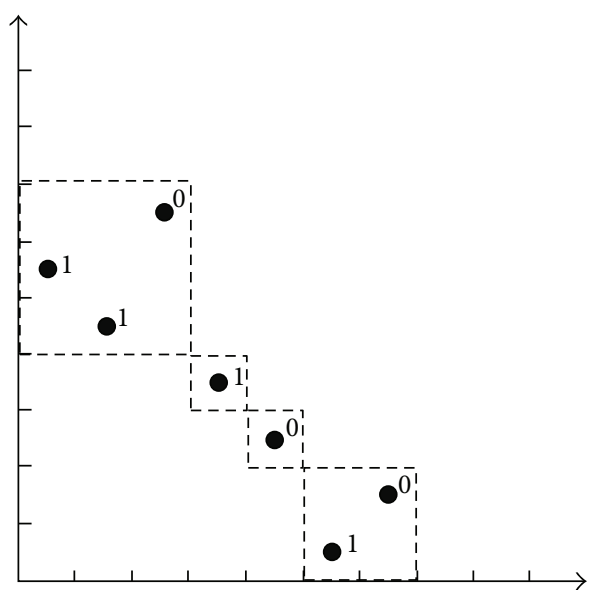

FIGURE 2: Matrix representation of $\sigma=\left(\begin{array}{lllllll}6 & 5 & 7 & 4 & 3 & 1 & 2\end{array}\right.$, $\begin{array}{llllllll}1 & 1 & 0 & 1 & 0 & 1 & 0 \text { ) with reverse irreducible blocks outlined. }\end{array}$

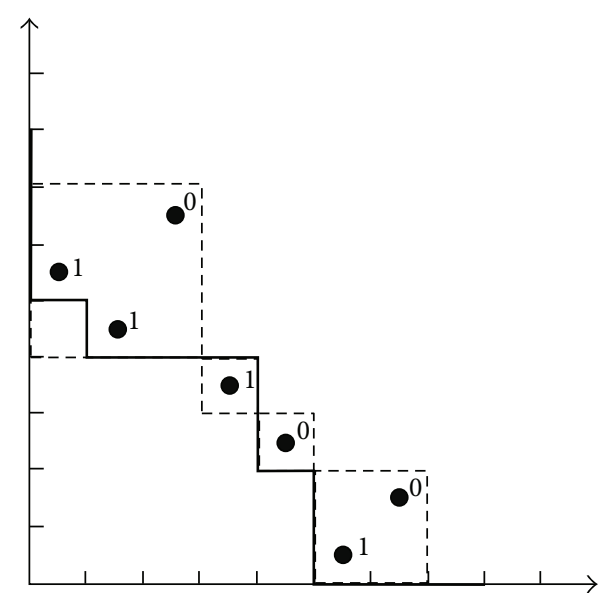

FIgURE 3: The Dyck path corresponding to $\sigma=$ $\left(\begin{array}{lllllllllllll}6 & 5 & 7 & 4 & 3 & 1 & 2,1 & 1 & 0 & 1 & 0 & 1 & 0\end{array}\right)$ added to Figure 2.

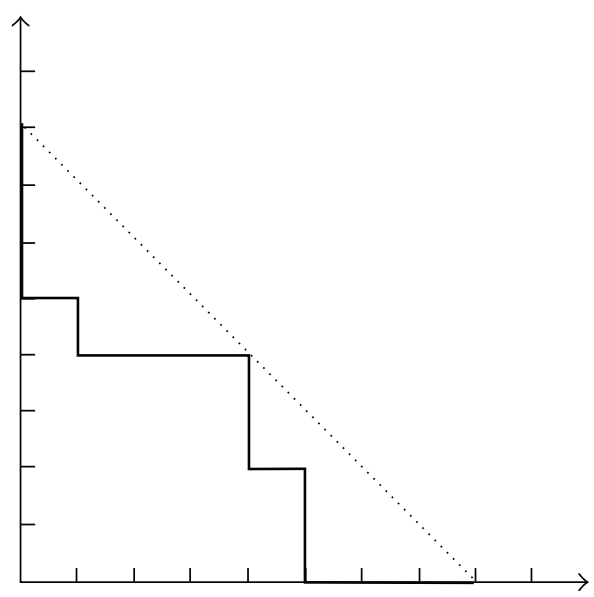

FIGURE 4: The Dyck path corresponding to $\sigma=\begin{array}{llll}6 & 5 & 7 & 4\end{array}$ $\left.\begin{array}{lllllllll}3 & 1 & 2,1 & 1 & 0 & 1 & 0 & 1 & 0\end{array}\right)$. Note that the path only touches the line $y=-x+n+1$ after a singleton colored 1 .

Theorem 19 (see [8]). If $\tau=j \cdots 2 \quad 1$, where $j \geq 2$, then

$$
\begin{aligned}
& \sum_{n=0}^{\infty} \frac{t^{n}}{n !} \mid\left\{\sigma \in S_{n}: \tau \text {-mch }(\sigma)=0\right\} \mid \\
& =\left(\sum_{n=0}^{\infty} \frac{t^{j n}}{(j n) !}-\sum_{n=0}^{\infty} \frac{t^{j n+1}}{(j n+1) !}\right)^{-1} .
\end{aligned}
$$

Later Mendes and Remmel [9] refined this result by proving the following.

Theorem 20. When $\tau=j \cdots 2 \quad 1$,

$$
\begin{aligned}
\sum_{n=0}^{\infty} \frac{t^{n}}{n !} \sum_{\substack{\sigma \in S_{n} \\
\tau-\operatorname{mch}(\sigma)=0}} x^{\operatorname{des}(\sigma)} & \\
& =\frac{1}{1+\sum_{n=1}^{\infty} t^{n} / n ! \sum_{i \geq 0}(-1)^{i} \mathscr{R}_{n-1, i, j-1} x^{i}},
\end{aligned}
$$

where $\mathscr{R}_{n, i, j}$ is the number of rearrangements of $i$ zeroes and $n-i$ ones such that $j$ zeroes never appear consecutively.

The reason that Theorem 20 is a refinement of Theorem 19 is that Mendes and Remmel [9] proved that

$$
\sum_{i \geq 0}(-1)^{i} \mathscr{R}_{n, i, j-1}= \begin{cases}(-1)^{n} & \text { if } j \text { divides } n \\ (-1)^{n+1} & \text { if } j \text { divides } n+1 \\ 0 & \text { otherwise }\end{cases}
$$

so that setting $x=1$ in (87) yields (86).

The main goal of this section is to generalize Theorem 20 . That is, let $\tau^{(j)}=j(j-1) \cdots 1$ where $j \geq 3$. Then we shall consider three sets of patterns for $C_{k}<S_{n}$ :
(a) $\left(\tau^{(j)}, 0^{j}\right)$,
(b) $\left(\tau^{(j)}, j-1 \quad j-2 \cdots 10\right)$, 
(c) $\Upsilon$ which equals the set of all $\left(\tau^{(j)}, w\right)$ such that $w \in$ $\{0, \ldots, k-1\}^{j}, w$ is weakly decreasing, and $\operatorname{red}(w)=w$.

In [1], Kitaev et al. defined three different types of descents. That is, for elements $(\sigma, w) \in C_{k} 2 S_{n}$, they defined

$$
\begin{aligned}
& \operatorname{Des}((\sigma, w))=\left\{i: \sigma_{i}>\sigma_{i+1}, w_{i} \geq w_{i+1}\right\}, \\
& \operatorname{des}((\sigma, w))=|\operatorname{Des}((\sigma, w))|, \\
& \operatorname{WDes}((\sigma, w))=\left\{i: \sigma_{i}>\sigma_{i+1}, w_{i}=w_{i+1}\right\}, \\
& \operatorname{wdes}((\sigma, w))=|\operatorname{WDes}((\sigma, w))|, \\
& \operatorname{SDes}((\sigma, w))=\left\{i: \sigma_{i}>\sigma_{i+1}, w_{i}>w_{i+1}\right\}, \\
& \operatorname{sdes}((\sigma, w))=|\operatorname{SDes}((\sigma, w))| .
\end{aligned}
$$

We shall refer to $\operatorname{Des}((\sigma, w))$ as the descent set of $(\sigma, w)$, $\operatorname{WDes}((\sigma, w))$ as the weak descent set of $(\sigma, w)$, and $\operatorname{SDes}((\sigma, w))$ as the strict descent set of $(\sigma, w)$.

Theorem 21. Let $\tau^{(j)}=j \cdots 21$ where $j \geq 3$.

(1) If $u=0^{j}$, then

$$
\begin{aligned}
\sum_{n=0}^{\infty} \frac{t^{n}}{n !} \sum_{\substack{(\sigma, w) \in C_{k} S_{n} \\
(\tau, u)-\operatorname{mch}((\sigma, w))=0}} x^{\operatorname{wdes}((\sigma, w))} \\
\quad=\left(1+\sum_{n=1}^{\infty} \frac{t^{n}}{n !} k \sum_{i \geq 0}(-1)^{i} \mathscr{R}_{n-1, i, j-1} x^{i}\right)^{-1} .
\end{aligned}
$$

(2) If $u=j-1 \quad j-2 \cdots 21$, then

$$
\begin{aligned}
\sum_{n=0}^{\infty} \frac{t^{n}}{n !} \sum_{\substack{(\sigma, w) \in C_{k}<S_{n} \\
(\tau, u)-\operatorname{mch}((\sigma, w))=0}} x^{\operatorname{sdes}((\sigma, w))} \\
=\left(1+\sum_{n=1}^{\infty} \frac{t^{n}}{n !}\left(\begin{array}{l}
k \\
n
\end{array}\right) \sum_{i \geq 0}(-1)^{i} \mathscr{R}_{n-1, i, j-1} x^{i}\right)^{-1} .
\end{aligned}
$$

(3) If $\Upsilon$ equals the set of all $\left(\tau^{(j)}, w\right)$ such that $w \in$ $\{0, \ldots, k-1\}^{j}, w$ is weakly decreasing, and red $(w)=w$, then

$$
\begin{aligned}
\sum_{n=0}^{\infty} \frac{t^{n}}{n !} \sum_{\substack{(\sigma, w) \in C_{k} k S_{n} \\
\Upsilon-\operatorname{mch}((\sigma, w))=0}} x^{\operatorname{des}((\sigma, w))} \\
\quad=\left(1+\sum_{n=1}^{\infty} \frac{t^{n}}{n !}\left(\begin{array}{c}
k+n-1 \\
n
\end{array}\right) \sum_{i \geq 0}(-1)^{i} \mathscr{R}_{n-1, i, j-1} x^{i}\right)^{-1} .
\end{aligned}
$$

Proof. Our proof is an adaptation of the proof that Mendes and Remmel [9] used to prove Theorem 20. The basic idea is to show that (90), (91), and (92) arise by applying appropriate ring homomorphisms, defined on the ring $\Lambda$ of symmetric functions over infinitely many variables $x_{1}, x_{2}, \ldots$, to a simple symmetric function identity.

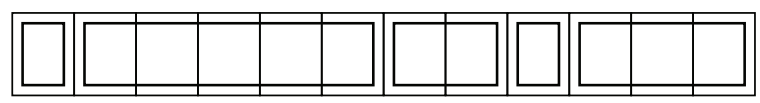

Figure 5: A brick tabloid of shape (1 2) and type $(1,1,2,3,5)$.

The $n$th elementary symmetric function, $e_{n}$, and the $n$th homogenous symmetric function, $h_{n}$, are defined by the generating functions

$$
\begin{aligned}
& E(t)=\sum_{n \geq 0} e_{n} t^{n}=\prod_{i}\left(1+x_{i} t\right), \\
& H(t)=\sum_{n \geq 0} h_{n} t^{n}=\prod_{i} \frac{1}{1-x_{i} t} .
\end{aligned}
$$

Clearly,

$$
H(t)=\frac{1}{E(-t)} .
$$

Let $\lambda=\left(\lambda_{1}, \ldots, \lambda_{\mathfrak{l}}\right)$ be an integer partition; that is, $\lambda$ is a finite sequence of weakly increasing positive integers. Let $\ell(\lambda)=\ell$ denote the number of parts of $\lambda$. If the sum of these integers is $n$, we say that $\lambda$ is a partition of $n$ and write $\lambda \vdash n$. For any partition $\lambda=\left(\lambda_{1}, \ldots, \lambda_{\ell}\right)$, let $e_{\lambda}=e_{\lambda_{1}} \cdots e_{\lambda_{\ell}}$. The wellknown fundamental theorem of symmetric functions says that $\left\{e_{\lambda}\right.$ : is a partition $\}$ is a basis for $\Lambda$ or that $\left\{e_{0}, e_{1}, \ldots\right\}$ is an algebraically independent set of generators for $\Lambda$. Similarly, if we define $h_{\lambda}=h_{\lambda_{1}} \cdots h_{\lambda_{\mathrm{p}}}$, then $\left\{h_{\lambda}\right.$ : is a partition $\}$ is also a basis for $\Lambda$.

Since the elementary symmetric functions $e_{\lambda}$ and the homogeneous symmetric functions $h_{\lambda}$ are both bases for $\Lambda$, it makes sense to talk about the coefficient of the homogeneous symmetric functions when written in terms of the elementary symmetric function basis. These coefficients have been shown to equal the sizes of certain sets of combinatorial objects up to a sign. A brick tabloid of shape $(n)$ and type $\lambda=\left(\lambda_{1}, \ldots, \lambda_{k}\right)$ is a filling of a row of $n$ squares of cells with bricks of lengths $\lambda_{1}, \ldots, \lambda_{k}$ such that bricks do not overlap. One brick tabloid of shape (12) and type $(1,1,2,3,5)$ is displayed In Figure 5.

Let $\mathscr{B}_{\lambda, n}$ denote the set of all $\lambda$-brick tabloids of shape $(n)$ and let $B_{\lambda, n}=\left|\mathscr{B}_{\lambda, n}\right|$. Through simple recursions stemming from (94), Eğecioğlu and Remmel [10] proved that

$$
h_{n}=\sum_{\lambda \vdash n}(-1)^{n-\ell(\lambda)} B_{\lambda, n} e_{\lambda} .
$$

We now consider three different ring homomorphisms which map $\Lambda$ to $\mathbb{Q}[x]$ where $\mathbb{Q}$ is the field of rational numbers. Since $e_{0}, e_{1}, \ldots$ is an algebraically independent set of generators for $\Lambda$, we can define a ring homomorphism by simply specifying its value on $e_{n}$ for all $n \geq 0$. For $n \geq 1$, define

$$
\begin{aligned}
& \Gamma_{1}\left(e_{n}\right)=\frac{(-1)^{n}}{n !} k \sum_{i \geq 0}(-1)^{n-i} \mathscr{R}_{n-1, i, j-1} x^{i}, \\
& \Gamma_{2}\left(e_{n}\right)=\frac{(-1)^{n}}{n !}\left(\begin{array}{c}
k \\
n
\end{array}\right) \sum_{i \geq 0}(-1)^{n-i} \mathscr{R}_{n-1, i, j-1} x^{i}, \\
& \Gamma_{3}\left(e_{n}\right)=\frac{(-1)^{n}}{n !}\left(\begin{array}{c}
k+n-1 \\
n
\end{array}\right) \sum_{i \geq 0}(-1)^{n-i} \mathscr{R}_{n-1, i, j-1} x^{i} .
\end{aligned}
$$


Our first goal is to prove the following facts.

(A) If $u=0^{j}$, then

$$
n ! \Gamma_{1}\left(h_{n}\right)=\sum_{\substack{(\sigma, w) \in C_{k} 2 S_{n} \\(\tau, u)-\operatorname{mch}((\sigma, w))=0}} x^{\mathrm{wdes}((\sigma, w))} .
$$

(B) If $u=j-1 j-2 \cdots 10$, then

$$
n ! \Gamma_{2}\left(h_{n}\right)=\sum_{\substack{(\sigma, w) \in C_{k}{ }^{2} S_{n} \\(\tau, u)-\operatorname{mch}((\sigma, w))=0}} x^{\operatorname{sdes}((\sigma, w))} .
$$

(C) If $\Upsilon$ consists of the set of all $\left(\tau^{(j)}, w\right)$ such $w \in$ $\{0, \ldots, k-1\}^{j}, w$ is weakly decreasing, and $\operatorname{red}(w)=w$, then

$$
n ! \Gamma_{3}\left(h_{n}\right)=\sum_{\substack{(\sigma, w) \in C_{k} S_{n} \\ \Upsilon-\mathrm{mch}((\sigma, w))=0}} x^{\operatorname{des}((\sigma, w))}
$$

Let $f(1, n)=k, f(2, n)=\left(\begin{array}{l}k \\ n\end{array}\right)$, and $f(3, n)=\left(\begin{array}{c}k+n-1 \\ n\end{array}\right)$. Then for $i \in\{1,2,3\}$,

$$
\begin{aligned}
n ! \Gamma_{i}\left(h_{n}\right)= & n ! \sum_{\lambda \vdash n}(-1)^{n-\ell(\lambda)} B_{\lambda, n} \Gamma_{i}\left(e_{\lambda}\right) \\
= & n ! \sum_{\lambda \vdash n}(-1)^{n-\ell(\lambda)} B_{\lambda, n} \prod_{m=1}^{\ell(\lambda)} \frac{(-1)^{\lambda_{m}}}{\lambda_{m} !} \\
& \times f\left(i, \lambda_{m}\right)\left(\sum_{i \geq 0}(-1)^{\lambda_{m}-i} \mathscr{R}_{\lambda_{m}-1, i, j-1} x^{i}\right) \\
= & \sum_{\lambda \vdash n} \sum_{B=\left(b_{1}, \ldots, b_{\ell(\lambda)}\right) \in \mathscr{B}_{\lambda, n}}\left(b_{1}, \ldots, b_{\ell(\lambda)}\right)(-1)^{\ell(\lambda)} \\
& \times \prod_{m=1}^{\ell(\lambda)} f\left(i, b_{m}\right)\left(\sum_{i \geq 0}(-1)^{b_{m}-i} \mathscr{R}_{b_{m}-1, i, j-1} x^{i}\right) .
\end{aligned}
$$

We wish to give a combinatorial interpretation of righthand side of (100). First we select a partition $\lambda \vdash n$ and then we select a brick tabloid $B=\left(b_{1}, \ldots, b_{\ell(\lambda)}\right) \in \mathscr{B}_{\lambda, n}$. Next we interpret the multinomial coefficient $\left(\begin{array}{c}n \\ b_{1}, \ldots, b_{\ell(\lambda)}\end{array}\right)$ as the number of ways to pick sets $S_{1}, \ldots, S_{\ell(\lambda)}$ of sizes $b_{1}, \ldots, b_{\ell(\lambda)}$, respectively, which partition $\{1, \ldots, n\}$. We then place these elements in $S_{m}$ in the bottom of the cells of the brick $b_{m}$ in decreasing order reading from left to right. Next if $i=1$, then $f\left(1, b_{m}\right)=k$ which we interpret as the ways of picking an element $i \in\{0, \ldots, k-1\}$ and placing $i$ at the top of

\begin{tabular}{|c|c|c|c|c|c|c|c|c|c|c|c|}
\hline$x$ & $x$ & -1 & $x$ & 1 & $x$ & 1 & $x$ & -1 & -1 & $\lambda$ & 1 \\
\hline 5 & 4 & 2 & 1 & 0 & 5 & 2 & & 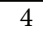 & 3 & & 0 \\
\hline 12 & 9 & 7 & 5 & 4 & 10 & 6 & 11 & 8 & 3 & 2 & 1 \\
\hline
\end{tabular}
each cell of $b_{m}$. If $i=2$, then $f\left(2, b_{m}\right)=\left(\begin{array}{c}k \\ b_{m}\end{array}\right)$ which we

\begin{tabular}{|c|c|c|c|c|c|c|c|c|c|c|c|}
\hline$x$ & $x$ & -1 & $x$ & 1 & $x$ & 1 & $x$ & -1 & -1 & $x$ & 1 \\
\hline 5 & 5 & 2 & 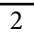 & 2 & 5 & 7 & 2 & 1 & 1 & & \\
\hline 12 & 9 & 7 & 5 & 4 & 10 & 6 & 11 & 8 & 3 & 2 & 1 \\
\hline
\end{tabular}
interpret as the ways of picking a strictly decreasing sequence $s=s_{1}>\cdots>s_{b_{m}}$ from $\{0, \ldots, k-1\}$ and placing $s_{j}$ on top of the $j$ th cell of $b_{m}$. If $i=3$, then $f\left(3, b_{m}\right)=\left(\begin{array}{c}k+b_{m}-1 \\ b_{m}\end{array}\right)$ which we interpret as the ways of picking a weakly decreasing sequence $s=s_{1} \geq \cdots \geq s_{b_{i}}$ from $\{0, \ldots, k-1\}$ and

\begin{tabular}{|c|c|c|c|c|c|c|c|c|c|c|c|c|}
$x$ & $x$ & -1 & $x$ & 1 & $x$ & 1 & $x$ & -1 & -1 & $x$ & 1 \\
\hline \hline 1 & 1 & 1 & 1 & 1 \\
12 & 9 & 7 & 5 & 4 \\
\hline
\end{tabular}

FIgURE 6: Filled labeled brick tabloids in $F_{12, i}$.

placing $s_{j}$ on top of the $j$ th cell of $b_{i}$. Finally, we interpret the term $\left(\sum_{i \geq 0}(-1)^{b_{m}-i} \mathscr{R}_{b_{m}-1, i, j-1} x^{i}\right)$ as follows. First, we pick a sequence $t=t_{1} \cdots t_{b_{m}-1}$ which consists of $i x$ 's and $b_{m}-$ $i-10$ 's for some $i$ such that there is no consecutive sequence of $j-1 x^{\prime} s$ in $t$. Then if $t_{s}=x$, we label the sth cell of $b_{m}$ with $x$, and if $t_{s}=0$, we label the sth cell of $b_{m}$ with -1 . Finally, we label the last cell of $b_{m}$ with -1 . Thus for any given $i$, we will have $b_{m}-i$ cells labeled with -1 which accounts for the sign $(-1)^{b_{i}-1}$. The only term in (100) that we have not accounted for is the term $(-1)^{\ell(\lambda)}$ which we use to change that last cell of each brick from -1 to 1 . We shall call an object created in this way a filled labeled brick tabloid and we let $\mathscr{F}_{n, i}$ denote the set of all filled labeled brick tabloids that arise in this way for interpreting $n ! \Gamma_{i}\left(h_{n}\right)$. Thus a $C \in \mathscr{F}_{n, i}$ consists of a brick tabloid $T$, a permutation $\sigma \in S_{n}$, a sequence $w \in\{0, \ldots, k-1\}^{n}$, and a labeling $L$ of the cells of $T$ with elements from $\{x, 1,-1\}$ such that the following conditions hold.

(a) $\sigma$ is strictly decreasing in each brick.

(b) (1) If $i=1$, then $w$ is constant in each brick.

(2) If $i=2$, then $w$ is strictly decreasing in each brick.

(3) If $i=3$, then $w$ is weakly decreasing in each brick.

(c) The final cell of each brick is labeled with 1 .

(d) Each cell which is not a final cell of a brick is labeled with $x$ or -1 and there is no consecutive string of cells of length $j-1$ labeled $x$ within a brick.

We then define the weight $w(C)$ of $C$ to be the product of all the $x$ labels in $L$ and the sign $\operatorname{sgn}(C)$ of $C$ to be the product of all the -1 labels in $L$.

For example, if $n=12, k=6, j=4$, and $T=(5,2,5)$, then Figure 6 pictures a filled labeled brick tabloid $C \in \mathscr{F}_{12,1}$ at the top, a a filled labeled brick tabloid $C \in \mathscr{F}_{12,2}$ in the middle, and a filled labeled brick tabloid $C \in \mathscr{F}_{12,3}$ at the bottom. In each case, $w(C)=x^{6}$ and $\operatorname{sgn}(C)=-1$.

Next we define sign-reversing, weight-preserving involutions $I_{i}$ on $F_{n, i}$. To define $I_{i}(C)$, we scan the cells of $C=$ 


\begin{tabular}{|c|c|c|c|c|c|c|c|c|c|c|c|}
\hline$x$ & $x$ & & $x$ & 1 & $x$ & 1 & $x$ & -1 & -1 & $x$ & 1 \\
\hline 1 & 1 & 1 & 1 & 1 & 4 & 4 & 0 & 0 & 0 & 0 & 0 \\
\hline 12 & 9 & 7 & 5 & 4 & 10 & 6 & 11 & 8 & 3 & 2 & 1 \\
\hline
\end{tabular}

\begin{tabular}{|c|c|c|c|c|c|c|c|c|c|c|c|}
\hline$x$ & $x$ & & $x$ & 1 & $x$ & 1 & $x$ & -1 & -1 & $x$ & 1 \\
\hline 5 & 4 & 2 & 1 & 0 & 5 & 2 & 5 & 4 & 3 & 1 & 0 \\
\hline 12 & 9 & 7 & 5 & 4 & 10 & 6 & 11 & 8 & 3 & 2 & 1 \\
\hline
\end{tabular}

\begin{tabular}{|c|c|c|c|c|c|c|c|c|c|c|c|}
\hline$x$ & $x$ & & $x$ & 1 & $x$ & 1 & $x$ & -1 & -1 & $x$ & 1 \\
\hline 5 & 5 & 2 & 2 & 2 & 5 & 0 & 2 & 1 & 1 & 1 & 0 \\
\hline 12 & 9 & 7 & 5 & 4 & 10 & 6 & 11 & 8 & 3 & 2 & 1 \\
\hline
\end{tabular}

Figure 7: $I_{i}(C)$ for the $C$ s in Figure 6.

(T, $\sigma, w, L)$ from left to right looking for the leftmost cell $t$ such that either

(i) $t$ is labeled with -1 ,

(ii) $t$ is at the end of a brick $b_{j}$ and the brick $b_{j+1}$ immediately following $b_{j}$ has the property that $\sigma$ is strictly decreasing in all the cells corresponding to $b_{j}$ and $b_{j+1}$ and $w$ is constant on all the cells corresponding to $b_{j}$ and $b_{j+1}$ if $i=1$,

$\cdot w$ is strictly decreasing on all the cells corresponding to $b_{j}$ and $b_{j+1}$ if $i=2$,

- $w$ is weakly decreasing on all the cells corresponding to $b_{j}$ and $b_{j+1}$ if $i=3$.

In case (i), $I_{i}(C)=\left(T^{\prime}, \sigma^{\prime}, w^{\prime}, L^{\prime}\right)$ where $T^{\prime}$ is the result of replacing the brick $b$ in $T$ containing $t$ by two bricks $b^{*}$ and $b^{* *}$ where $b^{*}$ contains the cell $t$ plus all the cells in $b$ to the left of $t$ and $b^{* *}$ contains all the cells of $b$ to the right of $t, \sigma=\sigma^{\prime}, w=w^{\prime}$, and $L^{\prime}$ is the labeling that results from $L$ by changing the label of cell $t$ from -1 to 1 . In case (ii), $I_{i}(C)=\left(T^{\prime}, \sigma^{\prime}, r^{\prime}, L^{\prime}\right)$ where $T^{\prime}$ is the result of replacing the bricks $b_{j}$ and $b_{j+1}$ in $T$ by a single brick $b, \sigma=\sigma^{\prime}, w=w^{\prime}$, and $L^{\prime}$ is the labeling that results from $L$ by changing the label of cell $t$ from 1 to -1 . Note that since the last cell in each brick is labeled with a 1 , when we combine the two bricks in case (ii), we cannot create a run of $j-1$ consecutive cells which are labeled $x$. If neither case (i) or case (ii) applies, then we let $I_{i}(C)=C$. For example, if we consider the C's in $\mathscr{F}_{12, i}$ pictured in Figure 6, then their corresponding images $I_{i}(C)$ are pictured in Figure 7.

It is easy to see that $I_{i}$ is a weight-preserving, signreversing involution, and hence $I_{i}$ shows that

$$
n ! \Gamma_{i}\left(h_{n}\right)=\sum_{C \in \mathscr{F}_{n, i}, I_{i}(C)=C} \operatorname{sgn}(C) w(C) .
$$

Thus we must examine the fixed points $C=(T, \sigma, w, L)$ of $I_{i}$. First there can be no -1 labels in $L$ so that $\operatorname{sgn}(C)=1$. Moreover, if $b_{j}$ and $b_{j+1}$ are two consecutive bricks in $T$ and $t$ is the last cell of $b_{j}$, then
(1) If $i=1$, it cannot be the case that $\sigma_{t}>\sigma_{t+1}$ and $w_{t}=$ $w_{t+1}$ since otherwise we could combine $b_{j}$ and $b_{j+1}$,

(2) if $i=2$, it cannot be the case that $\sigma_{t}>\sigma_{t+1}$ and $w_{t}>$ $w_{t+1}$ since otherwise we could combine $b_{j}$ and $b_{j+1}$, and

(3) if $i=3$, it cannot be the case that $\sigma_{t}>\sigma_{t+1}$ and $w_{t} \geq$ $w_{t+1}$ since otherwise we could combine $b_{j}$ and $b_{j+1}$.

Thus if $s$ is the last cell of a brick, then $s \notin \operatorname{WDes}(\sigma, w)$ if $i=1$, $s \notin \operatorname{SDes}(\sigma, w)$ if $i=2$, and $s \notin \operatorname{Des}(\sigma, w)$ if $i=3$. However, the label on a cell $t$ of any brick $b_{j}$ must be $x$ if $t$ is not the last cell of the brick and must be 1 if $t$ is the last cell of a brick. Moreover, if $t$ is not the last cell of a brick, then $\sigma_{t}>\sigma_{t+1}$ and $w_{t}=w_{t+1}$ if $i=1, w_{t}>w_{t+1}$ if $i=2, w_{t} \geq w_{t+1}$ if $i=3$. Thus if $t$ is not the last cell of a brick, then $t \in \operatorname{WDes}(\sigma, w)$ if $i=1, t \in \operatorname{SDes}(\sigma, w)$ if $i=2$, and $t \in \operatorname{Des}(\sigma, w)$ if $i=3$. It follows that $\operatorname{sgn}(C)=1$ and $w(C)=\operatorname{wdes}((\sigma, w))$ if $i=1$, $w(C)=\operatorname{sdes}((\sigma, w))$ if $i=2$, and $w(C)=\operatorname{wdes}((\sigma, w))$ if $i=3$. Then

$$
\begin{aligned}
& n ! \Gamma_{1}\left(h_{n}\right)=\sum_{\substack{(\sigma, w) \in C_{k} \backslash S_{n} \\
(\tau, u)-\operatorname{mch}((\sigma, w))=0}} x^{\operatorname{wdes}((\sigma, w))} \quad \text { if } i=1, \\
& n ! \Gamma_{2}\left(h_{n}\right)=\sum_{\substack{(\sigma, w) \in C_{k} S_{n} \\
(\tau, u)-\operatorname{mch}((\sigma, w))=0}} x^{\operatorname{sdes}((\sigma, w))} \quad \text { if } i=2, \\
& n ! \Gamma_{3}\left(h_{n}\right)=\sum_{\substack{(\sigma, w) \in C_{k} S_{n} \\
(\tau, u)-\operatorname{mch}((\sigma, w))=0}} x^{\operatorname{des}((\sigma, w))} \text { if } i=3 .
\end{aligned}
$$

Therefore (A), (B), and (C) hold.

We can now prove (90) by applying $\Gamma_{1}$ to (94). That is, we have $u=0^{j}$ and $\tau=j j-1 \cdots 2 \quad 1$, and then

$$
\begin{aligned}
\sum_{n=0}^{\infty} \frac{t^{n}}{n !} \sum_{\substack{(\sigma, w) \in C_{k} S_{n} \\
(\tau, u)-\operatorname{mch}(=) 0}} x^{\mathrm{wdes}((\sigma, w))} \\
\quad=\Gamma_{1}\left(\sum_{n=0}^{\infty} h_{n} t^{n}\right)=\frac{1}{\Gamma_{1}\left(\sum_{n=0}^{\infty} e_{n}(-t)^{n}\right)} \\
=\frac{1}{\left(1+\sum_{n=1}^{\infty}(-t)^{n} k(-1)^{n} / n ! \sum_{i \geq 0}(-1)^{n-i} \mathscr{R}_{n-1, i, j-1} x^{i}\right)^{-1}} \\
=\frac{1}{\left(1+\sum_{n=1}^{\infty} k t^{n} / n ! \sum_{i \geq 0}(-1)^{n-i} \mathscr{R}_{n-1, i, j-1} x^{i}\right)^{-1}} .
\end{aligned}
$$

Similarly, (91) can be proved by applying $\Gamma_{2}$ to (94) and (92) can be proved by applying $\Gamma_{3}$ to (94).

In fact, we can calculate $R_{n, i, j}$ in several cases. For instance, routine combinatorial techniques are able to show that $R_{n-1, i, 2}$ is $\left(\begin{array}{c}n-i \\ i\end{array}\right)$ whenever $0 \leq i \leq\lfloor n / 2\rfloor$. When $j=3$, we find that

$$
R_{n-1, i, 3}=\sum_{p=0}^{\lfloor i / 2\rfloor}\left(\begin{array}{c}
n+1-i \\
p, i-2 p, n+1
\end{array} 2 i+p\right) .
$$


We can also find a generating function for $R_{n, i, j}$ by considering the following argument. Take a rearrangement that is counted by $R_{n, i, j}$. Now remove the final number of the rearrangement. Either

(a) we have removed a 0 ,

(b) we have removed a 1 .

In case (a), we can count the number of such rearrangements by $R_{n-1, i-1, j}$, except that we have overcounted by those rearrangements counted by $R_{n-1, i-1, j}$ which end in $j-1$ zeroes. We can correct this by considering removing the last $j-1$ zeroes of the over-counted rearrangements, leaving those of length $n-j$ with $i-j$ zeros ending in a 1 , of which there are $R_{n-j-1, i-j, j}$. In case (b), we can count the number of such sequences by $R_{n-1, i, j}$. Thus we obtain the following relationship:

$$
R_{n, i, j}=R_{n-1, i-1, j}-R_{n-j-1, i-j, j}+R_{n-1, i, j}
$$

Next, we multiply by $q^{i} x^{n}$ and sum over all $n$, from which we obtain a generating function with a finite sum of small $R$ values in the numerator and a quotient of $1-x q-x+q^{i} x^{j+1}$.

\section{Acknowledgment}

J. Remmel was partially supported by NSF Grant DMS 0654060 and M. Riehl was supported by UWEC's Office of Research and Sponsored Programs.

\section{References}

[1] S. Kitaev, A. Niedermaier, J. B. Remmel, and A. Riehl, "Generalized pattern matching conditions for $C_{k}>S_{n}$ " ISRN Combinatorics, vol. 2013, Article ID 634823, 20 pages, 2013.

[2] E. S. Egge, "Restricted colored permutations and Chebyshev polynomials," Discrete Mathematics, vol. 307, no. 14, pp. 1792-1800, 2007.

[3] T. Mansour, "Pattern avoidance in coloured permutations," Séminaire Lotharingien de Combinatoire, vol. 46, article B46g, p. 12, 2001.

[4] T. Mansour, "Coloured permutations containing and avoiding certain patterns," Annals of Combinatorics, vol. 7, no. 3, pp. 349-355, 2003.

[5] T. Mansour and J. West, "Avoiding 2-letter signed patterns," Séminaire Lotharingien de Combinatoire, vol. 49, article B49a, p. 11, 2002.

[6] R. Simion, "Combinatorial statistics on type-B analogues of noncrossing partitions and restricted permutations," Electronic Journal of Combinatorics, vol. 7, no. 1, pp. 1-27, 2000.

[7] A. Duane and J. Remmel, "Minimal overlapping patterns in colored permutations," Electronic Journal of Combinatorics, vol. 18 , no. 2, p. 25, 2011.

[8] I. P. Goulden and D. M. Jackson, Combinatorial Enumeration, A Wiley-Interscience Publication, John Wiley \& Sons, New York, NY, USA, 1983.

[9] A. Mendes and J. Remmel, "Permutations and words counted by consecutive patterns," Advances in Applied Mathematics, vol. 37, no. 4, pp. 443-480, 2006.
[10] Ö. Eğecioğlu and J. B. Remmel, "Brick tabloids and the connection matrices between bases of symmetric functions," Discrete Applied Mathematics, vol. 34, no. 1-3, pp. 107-120, 1991. 


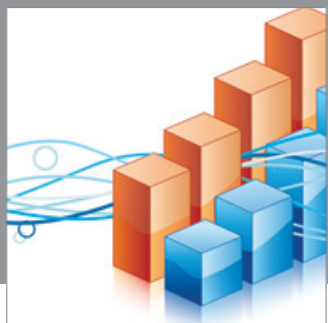

Advances in

Operations Research

mansans

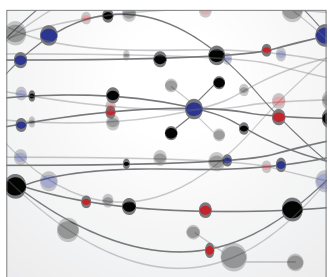

The Scientific World Journal
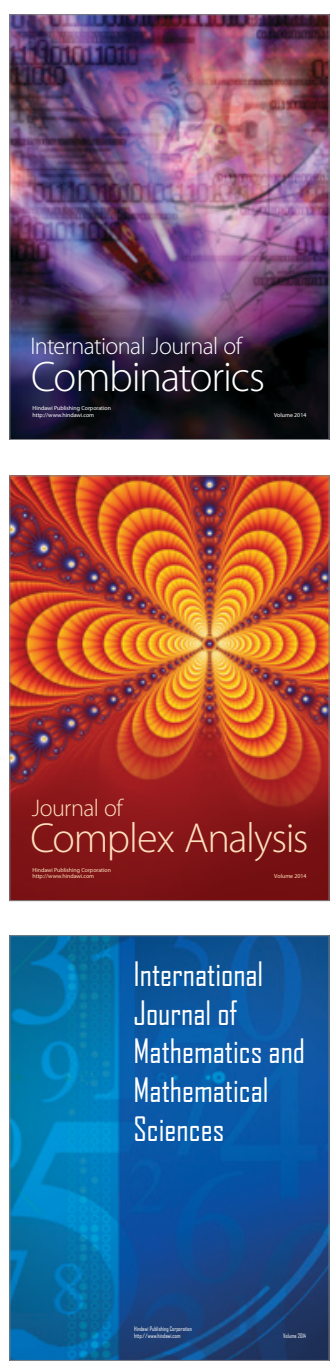
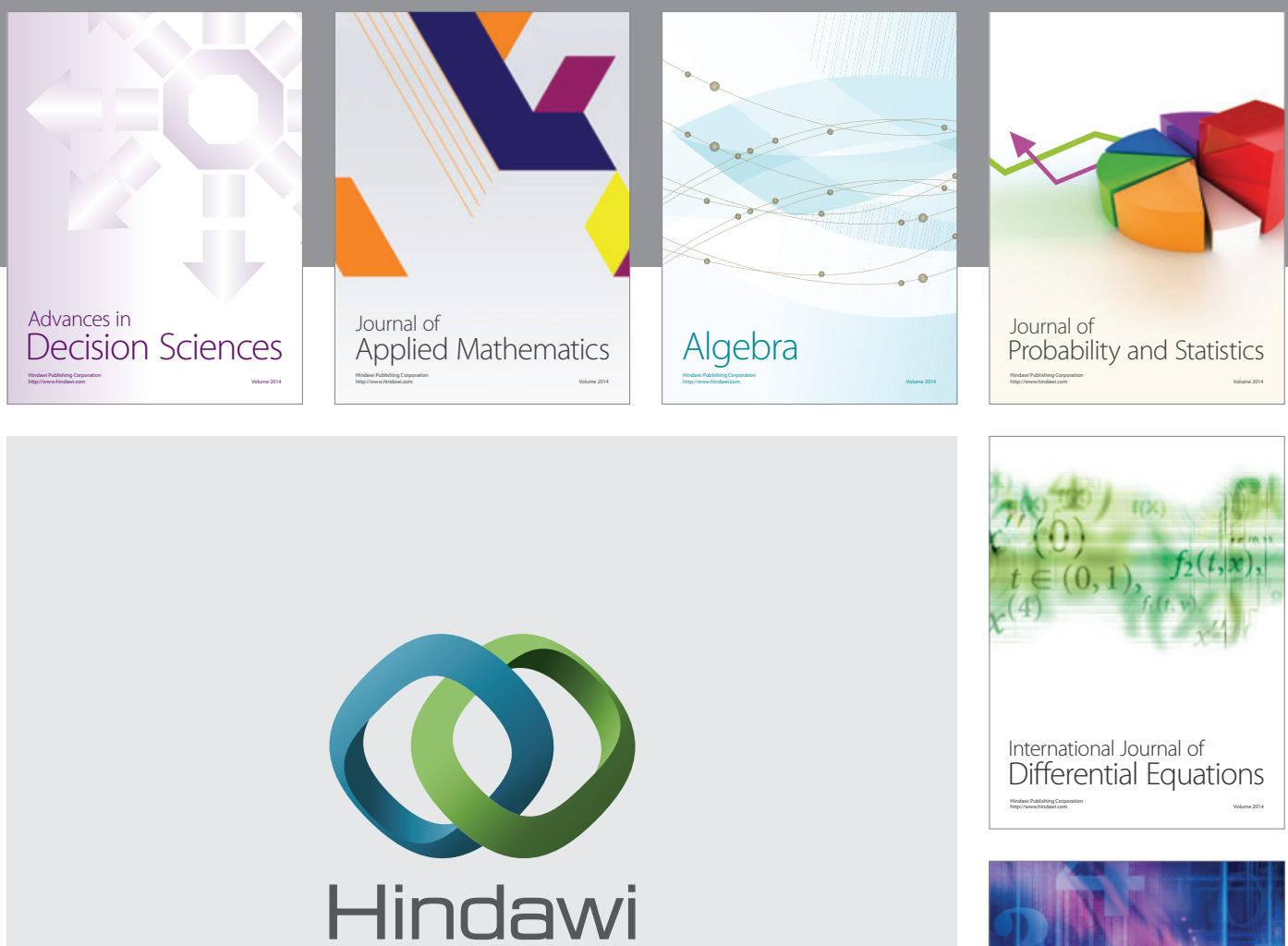

Submit your manuscripts at http://www.hindawi.com
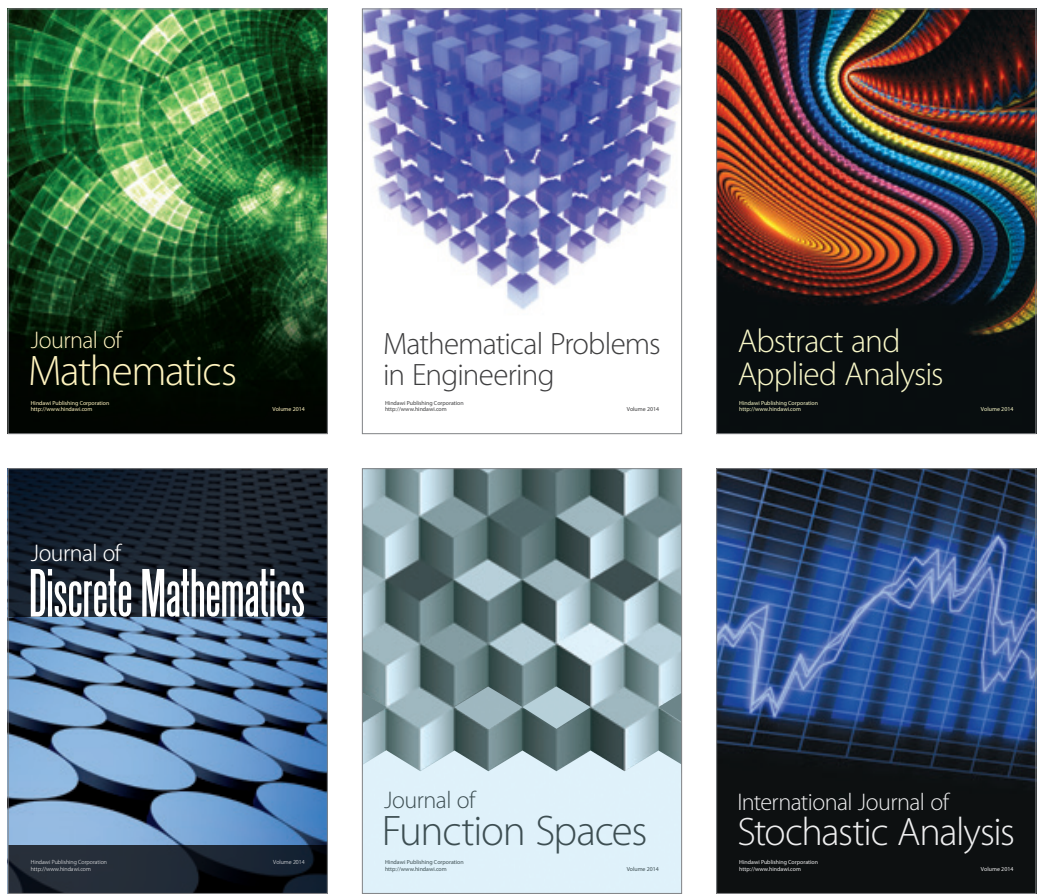

Journal of

Function Spaces

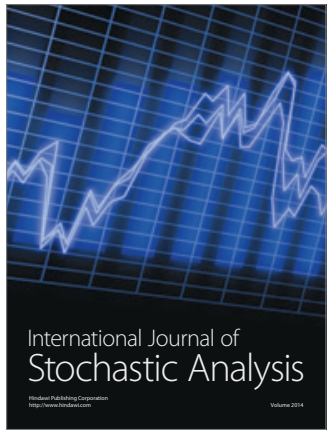

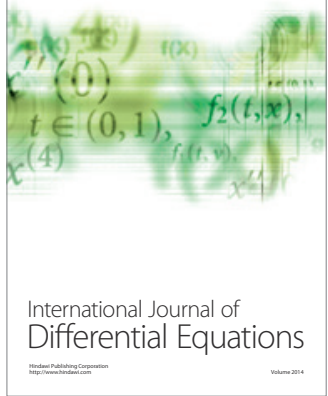
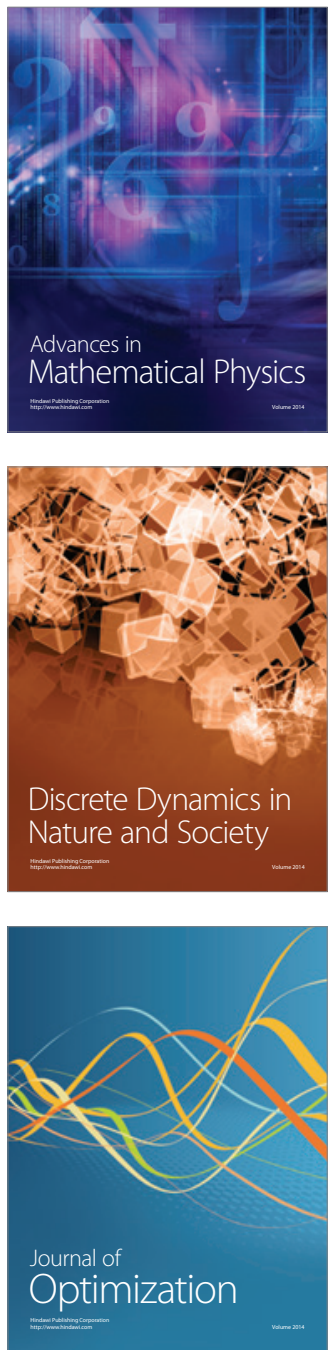